\title{
CFD-CAA Coupled Calculations of a Tandem Cylinder Configuration to Assess Facility Installation Effects
}

\author{
Stéphane Redonnet ${ }^{1}$ \\ ONERA (French Aerospace Centre), BP 72 - 29 av Division Leclerc, Châtillon, 92322, France \\ David P. Lockard ${ }^{2}$, Mehdi R. Khorrami ${ }^{3}$, Meelan M. Choudhari ${ }^{3}$ \\ NASA Langley Research Center, MS 128, Hampton, VA 23681-2199
}

This paper presents a numerical assessment of acoustic installation effects in the tandem cylinder (TC) experiments conducted in the NASA Langley Quiet Flow Facility (QFF), an open-jet, anechoic wind tunnel. Calculations that couple the Computational Fluid Dynamics (CFD) and Computational Aeroacoustics (CAA) of the TC configuration within the QFF are conducted using the CFD simulation results previously obtained at NASA LaRC. The coupled simulations enable the assessment of installation effects associated with several specific features in the QFF facility that may have impacted the measured acoustic signature during the experiment. The CFD-CAA coupling is based on CFD data along a suitably chosen surface, and employs a technique that was recently improved to account for installed configurations involving acoustic backscatter into the CFD domain. First, a CFD-CAA calculation is conducted for an 'isolated TC configuration' to assess the coupling approach, as well as to generate a reference solution for subsequent assessments of QFF installation effects. Direct comparisons between the CFD-CAA calculations associated with the various installed configurations allow the assessment of the effects of each component (nozzle, collector, etc.) or feature (confined vs. free jet flow, etc.) characterizing the NASA LaRC QFF facility.

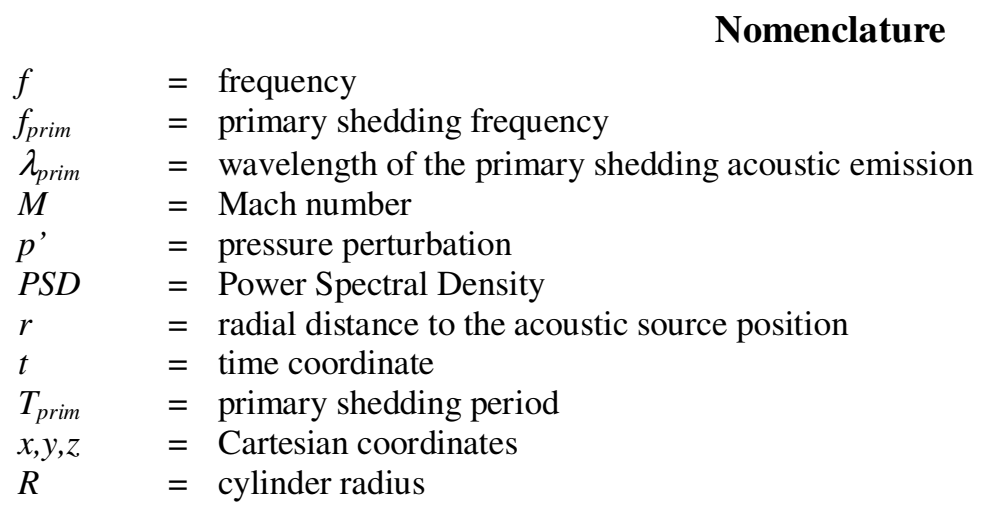

\section{Introduction}

he noise environment around airports is a major concern in the world, with many local communities exposed to
high levels of aircraft noise. Effective reduction of such 'noise pollution' represents an important environmental
challenge for the foreseeable future. The noise signature of an aircraft includes two main contributors; namely, the
engine noise component, which includes noise radiation from both turbomachinery (fan, turbine, combustion, etc.)
and the jet, as well as an airframe (or aerodynamic) noise component attributed to the interaction between turbulent
flow over the airframe and the adjacent solid structures, such as wings, slats, flaps, landing gears and cavities, etc.

\footnotetext{
${ }^{1} \mathrm{PhD}$, Research scientist, CFD and Aeroacoustics Department, ONERA.

${ }^{2}$ Research scientist, Computational AeroSciences Branch, Senior Member, AIAA.

${ }^{3}$ Research scientist, Computational AeroSciences Branch, Associate Fellow, AIAA.
} 
Although engine noise accounts for a dominant portion of the overall aircraft noise during take-off, the airframe noise component becomes equally important during the approach for landing, when the engine thrust is considerably reduced. In their effort to develop quieter airplanes, aircraft manufacturers need to design airframes that minimize the intensity of flow induced acoustic radiation to the far field. Consequently, the development of capabilities that offer both a deeper understanding and an accurate prediction of the physical phenomena related to aerodynamic noise generation and propagation mechanisms has became a priority for the aerospace research community. In particular, numerical simulations have emerged as a valuable complement to the more traditional experimental methods.

\section{Aerodynamic Noise Prediction and Hybrid Approaches}

Prediction of aerodynamic noise is a complex problem that includes physical phenomena over a broad range of spatio-temporal scales. More precisely, the generation of acoustic disturbances is driven by turbulent structures of high amplitude and small space-time correlations, while the propagation of the acoustic disturbances is characterized by waves of low amplitude and large space-time correlations. Although both phenomena are ruled by the same compressible Navier-Stokes equations, they cannot be easily predicted via a single calculation because the computational resources required to resolve all of the relevant scales would be far too high. To make the numerical approach tractable in a practical context, the overall aeroacoustic problem is therefore decomposed into a set of coupled subproblems that focus on individual subregions of the overall spatial domain. Each subproblem has a specific range of amplitudes and physical scales that can be addressed using a numerical method that is customized to the dominant physics in that subregion. Methods involving a mixture of techniques in this manner are classified as hybrid methods for aeroacoustic prediction.

In a general sense, aeroacoustic hybrid methods are comprised of three stages (see Fig. 1), which are respectively devoted to the 1) acoustic generation and near-field propagation, 2) mid-field propagation where the acoustic amplitudes are linear but the mean flow is non-uniform, and 3) far-field propagation over the majority of the distance from the source to the observer at ground level, wherein the mean flow is virtually uniform. The acoustic generation and early propagation (stage 1) can be simulated with a compressible unsteady CFD approach, whether it involves DNS (Direct Numerical Simulation), LES (Large Eddy Simulation), unsteady Reynolds averaged NavierStokes equations, or a judicious mix of these techniques. The acoustic far-field radiation (stage 3) can be easily predicted with an Integral Method (IM), such as the well-known Kirchhoff ${ }^{1}$ or FWH (Ffowcs Williams - Hawkings ${ }^{2}$ ) integral techniques. The acoustic mid-field propagation (stage 2), can be neglected under certain special circumstances such as an isolated jet. However, stage 2 cannot generally be ignored in airframe noise problems, because of the increased cost of extending the viscous, nonlinear CFD computations from stage 1 to include sufficient resolution of refraction across mean-flow non-uniformities and reflections by solid obstacles far from the region of acoustic generation. Although a Boundary Element Method (BEM) may be sufficient to model acoustic reflections, only a higher-fidelity computational aeroacoustics (CAA) approach can simultaneously account for both reflection and refraction effects. Such high-fidelity CAA approaches must resolve acoustic propagation over relatively large distances across a non-uniform mean flow, but does not need to account for turbulent fluctuations

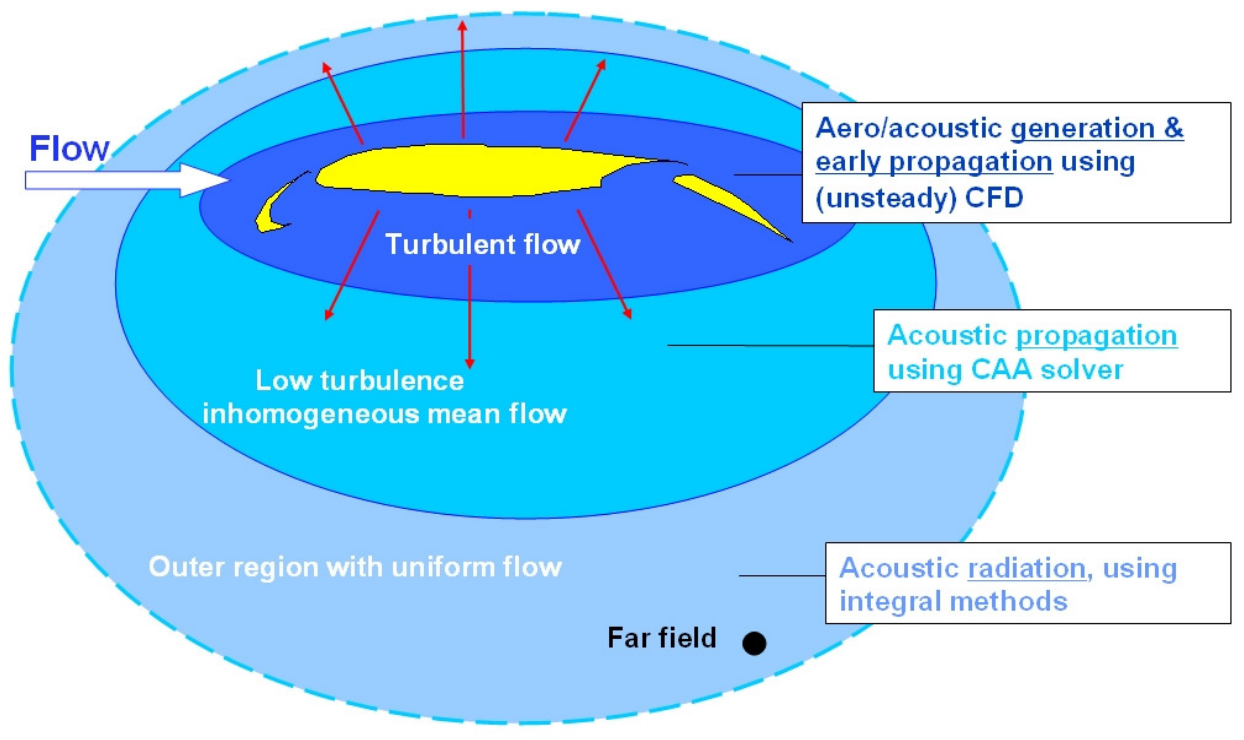

Figure 1. Three-step CAA hybrid approach

2

American Institute of Aeronautics and Astronautics 
and, perhaps, even viscous effects on acoustic propagation. This may typically be accomplished with the Euler equations or a linearized version thereof.

A critical aspect of developing such hybrid methodologies corresponds to the coupling, i.e., the information exchange, between the prediction modules for individual stages. The nature of this coupling is problem dependent, because of significant variations in the interdependencies between the various stages from one problem to another. However, except in problems involving acoustic feedback (e.g. screech tones), the coupling between the various stages is weak, i.e., primarily unidirectional ${ }^{3}$. Under this scenario, feedback from a given stage to the previous one can then be neglected.

Implementing an appropriate one-way coupling from the unsteady CFD to the CAA stage is an essential ingredient in hybrid aeroacoustic methods that seek to simulate the full cycle of events associated with a complex aerodynamic noise problem, ranging from near-field generation to far-field radiation and including the mid-field propagation. As discussed in Ref. 3, derivation of such coupling is a challenging task. In particular, such a coupling has to ensure that all acoustic events generated in stage 1 as part of the CFD simulation will be correctly transmitted to the CAA solver for stage 2 without any loss or duplication of the acoustic sources. Currently, at least two approaches for coupling CFD and CAA solvers exist, namely those based on 1) volumetric and 2) surface coupling.

Initially developed at $\mathrm{ONERA}^{4}$, the CFD-CAA surface coupling was successfully applied to several practical airframe noise applications ${ }^{5-7}$ such as acoustic emission by both an in-flight NACA0012 airfoil with a blunted trailing edge and a thick plate embedded within a flow. Very recently, the technique was improved further ${ }^{3}$ to deal with installed configurations; more precisely, the coupling process was modified to be transparent to the propagation of acoustic waves reflected back through the coupling interface - an event likely to occur whenever installed configurations are considered, since solid bodies surrounding the source region can reflect back anything the latter radiates. Such a CFD-CAA surface coupling technique was extensively used within the present effort, so as to enable coupled calculations of the noise emitted by a Tandem Cylinder configuration including installation effects.

\section{CFD-CAA Calculations of the Tandem Cylinder Configuration}

The present section is devoted to the Tandem Cylinder configuration and the results of applying the CFD/CAA surface coupling technique to this configuration.

\section{A. Background}

To better understand some of the generic physical mechanisms associated with landing gear noise sources, a combined experimental and computational campaign was carried out at NASA Langley Research Center, focusing on both the aerodynamics and the acoustics of a Tandem Cylinder (TC) configuration. The model geometry is defined by two identical cylinders, spatially separated in the streamwise direction of an incoming flow (see left side of Fig. 2). Such a simplified configuration constitutes an ideal test case for investigating the physics of aerodynamic noise associated with component interaction as well as validating high-fidelity numerical prediction tools for similar sources of noise in actual aircraft undercarriage systems. To this end, extensive experimental data has been
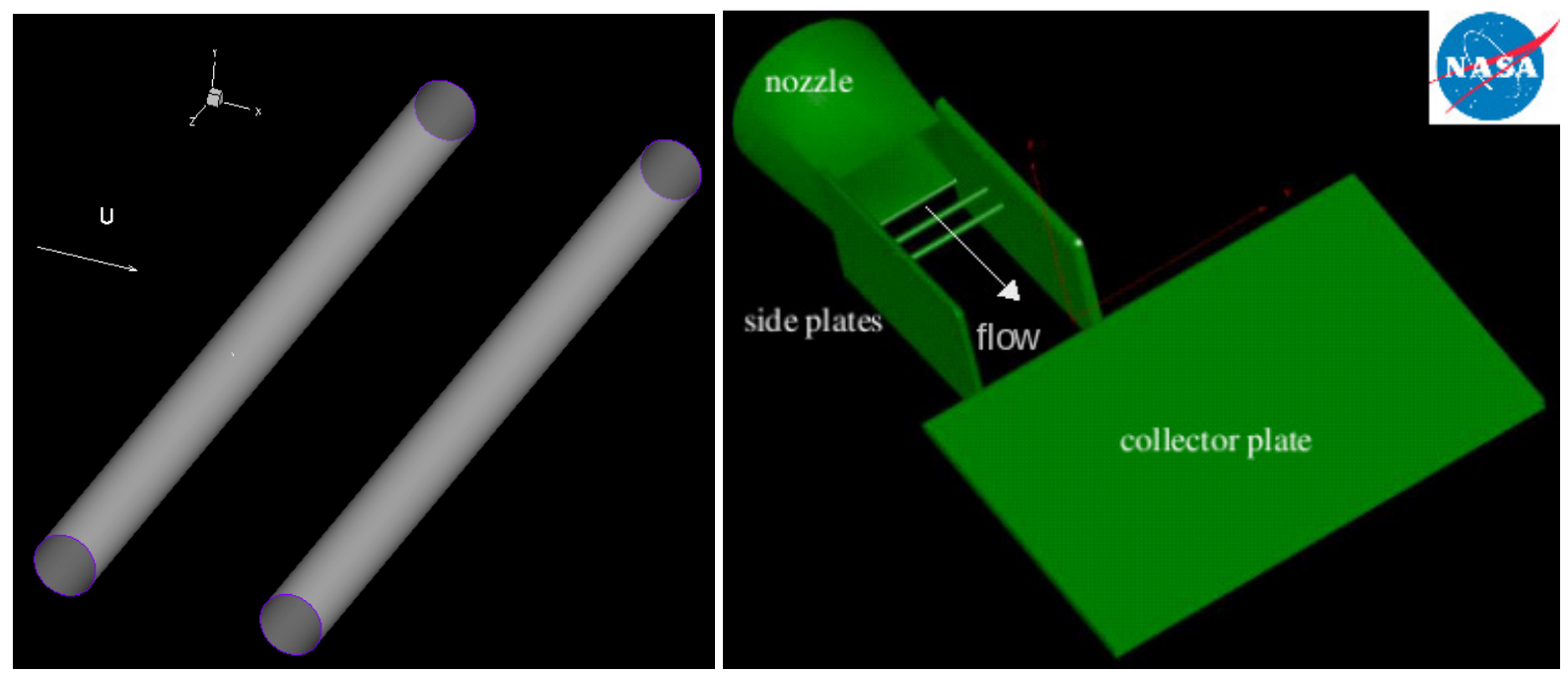

Figure 2: Tandem Cylinders, as isolated (left) and installed within NASA/LaRC QFF facility (right) 
collected $^{8-11}$ and compared to the results of 3-D, unsteady CFD computations ${ }^{12,13}$. Despite the favorable comparison between the measured and computed spectra, a legitimate concern existed about some of the obvious differences occurring between the installed TC configuration that had been tested and the simplified configuration that was computed. In other words, questions arose about the fact that these calculations did not incorporate any of the possible installation effects that could have occurred in the experiments. Indeed, as indicated previously, accounting for all or part of the installation components (e.g., QFF side plates) during the initial CFD stage would have been far too expensive, requiring a fine mesh to compute not only the cylinders, but also the side walls supporting / surrounding them. In addition, the intrinsic limitations of Helmoltz-based integral methods ${ }^{2}$ limit the FWH stage from correctly accounting for reflections / diffraction and the convection / refraction effects that may be caused by the QFF itself and its confined jet flow. Therefore, a numerical assessment of the acoustic reflection / diffraction effects of the main components of the anechoic facility (see right side of Fig. 2) was conducted a posteriori $^{14}$ with an Equivalent Source Model (ESM) approach and solver (Fast Scattering Code) ${ }^{15}$. There was, however, both a need and an interest to go further in such an a posteriori assessment of possible acoustic installation effects characterizing the TC acoustic experiments, by improving the fidelity of the acoustic propagation stage; first, it was desirable to account for the acoustic emission that had been effectively generated (or, at least, predicted by the CFD stage), rather than to model it via equivalent sources. Second, it seemed important to account not only for the reflection / diffraction effects induced by the experimental apparatus in the anechoic facility, but also for the (partial) convection / refraction effects characterizing its (confined and sheared) jet flow. These two requirements could obviously be fulfilled by a CFD-CAA coupled approach such as the one previously described, especially considering that its recent improvement enabled it to properly handle all acoustic propagation back through the coupling surface that was expected to occur in such an 'installed' situation. Consequently, several CFD-CAA coupled calculations of the installed TC configuration were conducted, all of which were 1) performed using the CFD-CAA surface coupling technique, and 2) based on the same CFD data ${ }^{12,13}$ (coming from the isolated TC computations initially performed at NASA LaRC). The near-field data was obtained using the second-order, NavierStokes CFD code CFL3D and a hybrid RANS/LES turbulence model. The computations were performed with a 13 million node grid with periodic boundary conditions in the spanwise direction. Only the geometry of the cylinders was included in the configuration. The span used in the computations was 18 cylinder diameters. For additional details on the simulations, the reader is referred to Refs. 12 and 13 .

\section{B. Preliminary Activities}

Before a CAA computation could be conducted, a number of preliminary (though non-trivial) tasks were required.

\section{III.B.1 CAA grid generation}

The first task consisted of generating proper CAA grids of the TC configuration, which had to be considered as both isolated and (partially) installed. Several meshes were thus derived via analytical means, all being targeted to capture correctly acoustic waves up to a frequency of $400 \mathrm{~Hz}$. Targeting such a (low) frequency was both justified by the characteristics of the primary shedding frequency $\left(f^{\prime}{ }_{\text {prim }}=220 \mathrm{~Hz}\right)$, and imposed by the sampling rate with which CFD simulation data had been stored. With a total of 28 blocks and comprised of 0.85 millions cells, the largest grid incorporated all the main features of the QFF (side plates, collector plate, jet nozzle - see Fig. 3), which were slightly approximated when the modifications would not adversely affect the accuracy of the simulations. First, the nozzle shape was simplified. That is, its slightly curved panels were replaced by straight ones. Then, considering their thickness could reasonably be neglected for such a low frequency source, both the nozzle and the side plates were modeled as infinitely thin. Finally, with the view of minimizing the CPU cost, the lateral $(z)$ dimension of

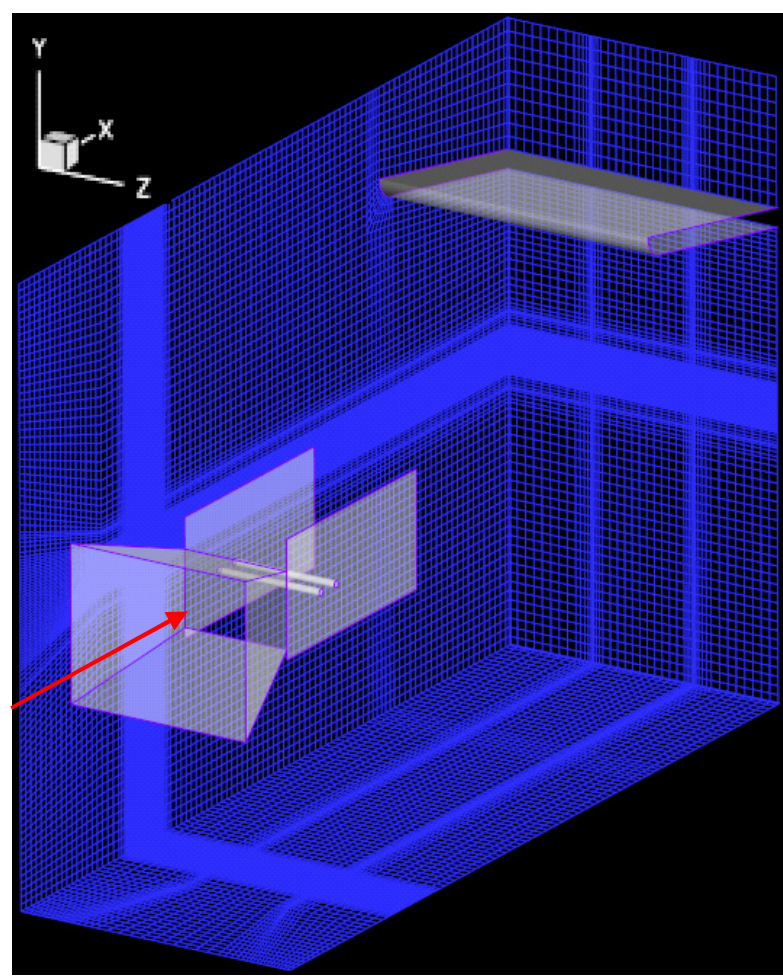

Figure 3. CAA mesh of the fullv installed TC 
the computational domain was restricted to include only $2 / 3^{\text {rd }}$ of the collector plate's spanwise extent. Given that the thickness of the collector had the potential to be non-negligible, the collector plate was meshed without approximation with special attention being paid to its rounded leading edge. The $x y$ plane computational domain was extended beyond the microphones, so that the CFD-CAA outputs could be directly compared to the CFD/FWH results initially obtained at those microphone locations ${ }^{12,13}$. Finally, in all the regions where a better spatial resolution was desired, the CAA grid density was locally and in a progressive manner refined. In particular, the grid in the source region, in the vicinity of jet shear layers, and around the plates' edges were made denser (by a factor of 7). Because the grid was generated analytically, smoothness of the grid and metrics could be assured to be greater than the order of the CAA solver. Although the grid was not systematically refined, the grid spacing was chosen to resolve the important range of frequencies in the problem based on the demonstrated resolving capability of the CAA code ${ }^{4,5,16}$. Furthermore, some grid sensitivity studies were performed, and the results were found to be relatively insensitive to refinement beyond the grid spacing used in this study.

\section{III.B.2 Derivation of both the mean flow and the CAA forcing}

A second preliminary task consisted of constructing the requisite base flow field (comprised of both the steady mean flow field for the perturbed Euler equations, and an associated instantaneous perturbed counterpart to be used in the forcing term in the CAA computation), before interpolating them onto the CAA grid. For this reconstruction, although available steady flow data (coming from RANS calculations) could have been used, the choice was made to generate a stationary field by time-averaging all the successive instantaneous CFD computed flow realizations that had been stored. This was dictated by a legitimate concern to preserve the formulation's consistency, which requires consistently defining both the mean and the perturbed quantities over the source region. Once the construction of the mean flow was achieved, all the fluctuating components corresponding to such a steady mean flow were calculated by subtracting the latter from the total field. Those decomposed mean and perturbed flow fields were then interpolated in space from the CFD to CAA grid using a (tri) linear process. Once interpolated onto the CAA grid, these steady mean and instantaneous perturbed flow fields had to be subjected to a few additional manipulations; first, since the CAA computational domain was extended beyond the CFD domain boundaries, the steady mean flow field was extrapolated and the missing data were algebraically reconstructed wherever needed. Then, the steady mean flow was smoothed out by successive applications of second order spatial filters. The main reason for doing so was to prevent insufficient grid resolution of the viscous mean flow gradients in the vicinity of the cylinders in the Euler-type CAA grid, which would have led to numerical instabilities. For the same reason, the boundary layers surrounding the cylinder surfaces were removed from the CAA mean flow, and a special treatment was applied to avoid large gaps between the values at the wall and the first point off the wall. The treatment involved overwriting the near wall values by extrapolating radially from the interior of the CAA domain, just outside the boundary layers .
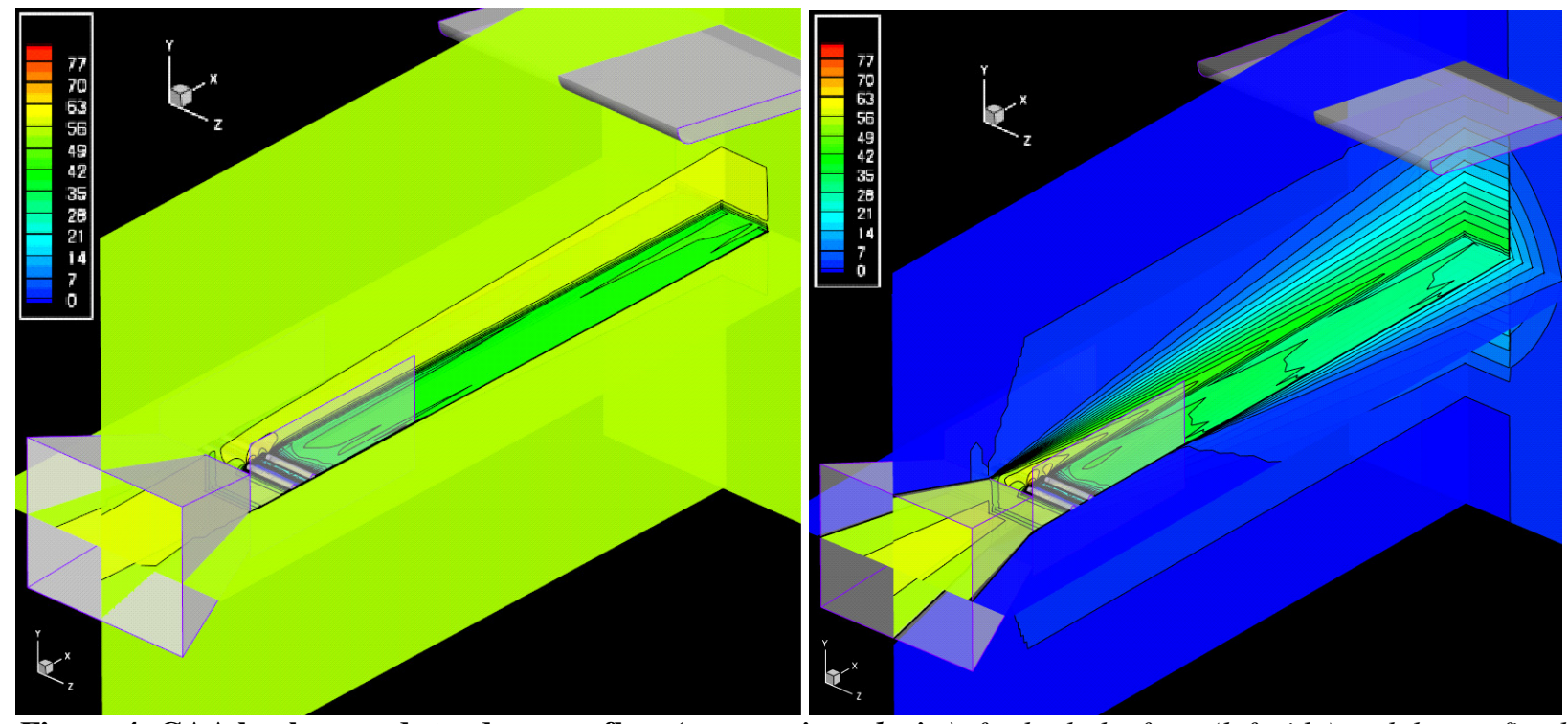

Figure 4: CAA background steady mean flow (streamwise velocity), for both the free-(left side) and the confined / sheared- jet (right side) 
Two different steady mean flows for the CAA calculation were derived; the first one was directly inherited from the CFD computational data set, providing a 'free-field' mean flow that matched the CFD steady values at infinity (for a free stream Mach number of 0.166 - see left side of Fig. 4). First, concerning the isolated TC configuration, such a free jet mean flow enabled a direct comparison with the CFD-FWH results, in order to validate further the coupling procedure itself. For the installed TC configuration, such a free-jet mean flow provided a reference solution against which results associated with a more realistic steady jet flow could be compared, allowing an assessment of convection / refraction effects of the QFF jet shear layers. One can notice that - as is - this extrapolated free jet flow was partly unrealistic, with a slightly non physical flow behavior occurring in the vicinity of both the nozzle and the collector. However, in the absence of a more suitable definition (which would have required performing a steady CFD calculation of the entire QFF configuration), and since no major issues were expected (just a few minor reflection / refraction errors), this background jet mean flow was used as is.

To improve the underlying physics, a second mean flow was derived with the aim of better representing the experimental conditions. In reality, the jet flow is initially confined within the nozzle walls. As the jet exits the nozzle into the ambient air, free shear layers develop at the nozzle lips, spreading out with axial distance. Acoustic waves can be refracted or deflected by such shear layers, modifying the directivity pattern. Similarly, the absence of any convection outside the jet may lead to propagation patterns that differ from the ones associated with the free-jet flow previously presented. Therefore, the latter was modified via analytical means using similarity functions to obtain a more realistic mean flow with jet spreading effects (see right side of Fig. 4). The functions were derived for spatial evolution at a rate driven by both 1) the x-axial distance to the nozzle exit plane and 2) the $\mathrm{y}-$ / z- lateral distance to the nozzle lips. The jet spreading rate was calibrated to match experimental observations in the QFF, corresponding to a spreading angle of $7^{\circ}$. Once properly derived, this 'confined / sheared' mean flow was further modified analytically to exhibit more realistic streamline patterns within the nozzle area.

The instantaneous perturbed flow field, having been interpolated in space between the CFD and CAA grids, was subjected to two additional manipulations. First, it was interpolated in time because the maximum time step compatible with the CAA grid was 35 times smaller than the one used for storing the CFD data. Although more advanced techniques could have been used, the data was linearly interpolated in time. This was achieved directly during the CAA calculation; the CAA solver internally reconstructed the temporal signals from two successive steps of CFD data. The second manipulation of the instantaneous perturbed flow field consisted of multiplying the forcing terms by a function that transitioned smoothly from unity around the cylinders to zero downstream of the rear cylinder. This was achieved in order to prevent the cylinder's wake from interacting with both 1) the aft part of the surface coupling interface and 2) the downstream boundary of the CAA domain.

Regardless of whether the steady mean flow was considered 'free' or 'confined / sheared,' the same set of instantaneous perturbed quantities was prescribed to be injected into the CAA calculation. This was done so that the acoustic source would be free of any bias possibly induced by different mean / perturbed splitting definitions. Furthermore, this was justified by theoretical considerations which indicate that as long as a perturbation problem remains linear, the fluctuating field is independent of the mean state (except for refraction effects). Finally, one can notice that from an aero/acoustic point of view, the Tandem Cylinders are neither fully isolated nor fully installed. Indeed, in order to simplify the treatment of the spanwise boundaries, the CFD employed periodic conditions, allowing aero/acoustic signals to be recycled in the spanwise direction. Consequently, the resulting perturbed fields could neither be considered as physically truncated nor bounded in the lateral direction - which would have been required for the CAA calculations to be formally isolated or installed. However, a CFD calculation of the installed configuration would have required excessive computer resources. Furthermore, the change in geometry could have altered the near-field source in an unpredictable manner making it difficult for the CAA to separate out the effects of reflection/refraction from that of an altered source. Therefore, the CFD $\rightarrow$ CAA forcing was truncated / bounded a posteriori, by limiting the injection region by the side plates in the spanwise direction (see Fig. 5). For the isolated configuration, the side plates were reduced as much as possible to extend just beyond the coupling region (see Fig. 7), because the presence of two narrow side plates was expected to have little effect on the initial propagation of acoustic waves.

\section{III.B.3 Location of the CFD /CAA surface coupling interface}

Finally, a third preliminary task was identifying the optimal location for the CFD-CAA surface coupling interface. Indeed, in general situations, the location of the coupling interface usually results from a compromise between the accuracy of the CFD calculation (with respect to the dissipation of acoustic waves) and the stability of the CAA calculation (instigated by spurious signals generated when nonlinear hydrodynamic flow features cross the interface ${ }^{3,5}$ ). In the present case, the CFD calculation was able to resolve the dominant part of the acoustic signal 
well into the near field because the bulk of the energy was at very low frequencies. However, using a coupling surface far from the cylinders would not have allowed the CAA computation to include the effects of reflection/diffraction from the side plates inside of the coupling surface. Consequently, for consistency more than for accuracy reasons, the coupling interface was located as close to the cylinders as possible without getting into regions of strong hydrodynamic activity (see Fig. 5) which would violate the assumption of linear disturbances underlying the hybrid methodology and also require a finer grid density in the CAA mesh to resolve viscous flow features ${ }^{3}$.

\section{Computed Cases and Set-Up \\ III.C.1 Computed cases}

For the first calculation the TC configuration was considered as isolated, i.e. free of any installation effects. This case provided a reference solution against which results obtained for the various installed configurations could be compared in order to assess the

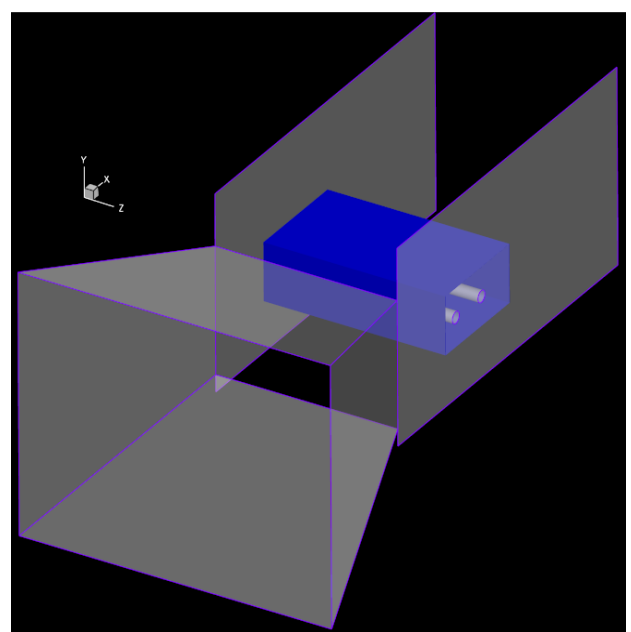

Figure 5: CFD-CAA surface coupling interface (in blue) specific reflection/diffraction and/or convection/refraction effects characterizing each configuration. Another objective was to validate further the CFD-CAA surface coupling approach, by comparing the results with the ones initially obtained by NASA LaRC researchers, who had computed the isolated TC case via a CFD/FWH approach $^{12,13}$. Obviously, for such an isolated case, the 'free jet' steady mean flow obtained from the CFD was imposed in the CAA calculation.

Additional calculations were performed for the installed configurations, first with the free-jet steady mean flow used for the isolated case (see left side of Fig. 4). Doing so was motivated by the desire to assess the reflection / diffraction effects independently from convection / refraction. To estimate separately the reflection / diffraction effects of each QFF component (side plates, collector plate, nozzle), additional calculations were conducted, with components added successively.

Finally, a last calculation focused on the more realistic case, i.e. the fully QFF-installed TC associated with the corresponding confined / sheared jet mean flow (see right side of Fig. 4). This calculation aimed not only at delivering still higher-fidelity results, but also at highlighting all possible convection / refraction effects characterizing the mean flow.

\section{III.C. 2 Computational set up}

All the CAA calculations were conducted with ONERA's sAbrinA.v0 solver $^{3-7,16-18}$, using NASA computational resources. sAbrinA.vO is a structured grid, time-accurate CAA code that solves the full Euler equations, either in a conservative form or non-conservative, perturbed form (with a splitting of the complete variables into a 'frozen' mean flow and a 'fluctuating' perturbation). The solver employs high-order, finite-difference operators, involving $6^{\text {th }}$-order spatial derivatives and $10^{\text {th }}$-order filters, as well as a $6^{\text {th }}$-order, multi-stage, Runge-Kutta timemarching scheme. The code deals with multi-block structured grids with one-to-one interfaces, and is fully parallelized using the Message Passing Interface (MPI) standard. Finally, the solver includes the usual boundary conditions (reflection by solid walls, non-reflecting, free-field radiation ${ }^{4,6}$, etc.), as well some unique to specific applications (such as the surface coupling technique that was extensively used in the present effort). The surface coupling technique consists of an implicit forcing of the CAA field, driven by instantaneous perturbed quantities previously obtained via analytical or numerical means (e.g. a CFD calculation). This

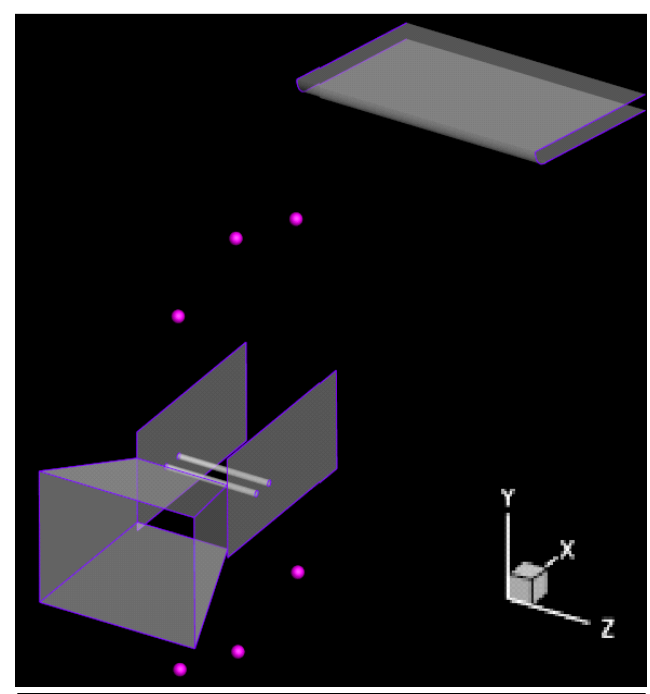

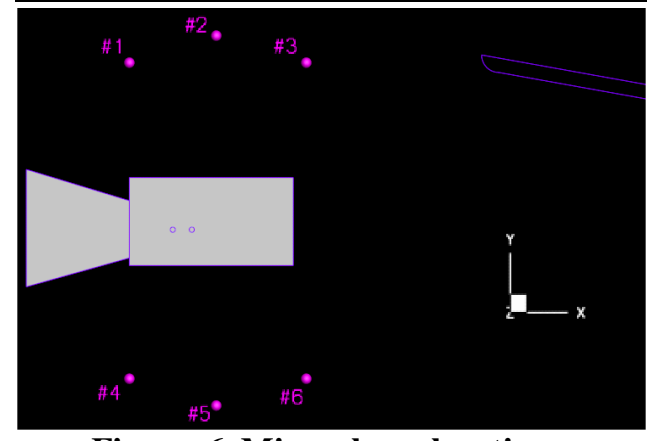

Figure 6. Microphone locations. 
technique has been described and validated in Ref 3. More detailed information about the sAbrinA.v0 solver and its underlying methodology can be found in Refs. 4 and 16.

All calculation cases were computed in parallel, via 64 processors on a PC cluster at NASA Langley Research Center. Calculations were run for a number of time steps covering the temporal extent of the CFD data record, corresponding to approximately 20 periods of the primary shedding frequency. The CAA grid and mean flow for each case were adjusted to properly model the effects of relevant components (i.e., scattering agents) within the QFF. The fully installed configuration used 28 domains, and only a subset of the 28 domains was used for the other cases involving fewer scattering components. As previously stated, the source from the CFD data imposed on the coupling surface in the CAA simulations was kept identical for all cases. The source distribution based on each stored time step of the CFD database was linearly interpolated to the appropriate time in the CAA simulation, and injected within the CAA computational domain via the surface coupling forcing. For all cases, the entire instantaneous perturbed pressure field was stored at regular intervals during the calculation. The perturbed pressure signal time history was also stored and post-processed (to obtain the power spectral density) at specific points corresponding to the microphone locations in the experiment (see Fig. 6). Finally, both the RMS (root mean square) perturbed pressure field and the acoustic phase field associated with the primary shedding frequency $\left(f^{\prime}\right.$ prim $)$ were determined from the computations.

\section{III.C.3 CPU performance}

Depending on the size of the CAA grid used for each case (between 530,000 cells for the isolated TC, and 853,000 cells for the fully installed TC), all calculations required between $11 \mathrm{~h}$ and $18 \mathrm{~h}$ of wall clock time on 64 CPUs. Scaled to the number of CAA iterations $(12,600)$ and cores (64) involved indicates an average CPU time of approximately $384 \mu \mathrm{sec} /$ iteration/cell/core. This (quite poor) CPU performance was mainly due to three factors; first, the I/O operations needed for both reading the CFD data and storing the CAA outputs were numerous and unoptimized. The performance penalty (of approximately $25 \%$ ) was accepted to allow greater flexibility in choosing what information to read and write. Secondly, these 0.5 to 0.8 million cell cases were not the best candidates for efficient distribution over 64 cores because there were twice as many ghost cells (requiring data exchanges across the network) as a serial computation. Although clearly not optimal, the parallel decomposition of the cases was nevertheless conducted, considering that it still resulted in a significant reduction in the time to solution. Finally, the Intel P4 2.53 CPUs used are now relatively old, and a more modern cluster can achieve significantly better performance; when run on 64-bit Itanium processors with a high-speed interconnect, similar calculations produced CPU times of approximately 50 $\mu \mathrm{sec} /$ iteration/cell/core.

\section{Isolated TC in a Free-Jet Flow}

The TC configuration was first considered as being as close to isolated as possible, i.e. free of any installation devices, with its steady mean flow being derived in a 'free-jet' sense.

\section{III.D.1 Calculation results and early analysis}

Figure 7 shows the instantaneous perturbed pressure field at a time $2 / 3 \mathrm{rd}$ of the way through the calculation. As one can observe in the $x y$ view corresponding to the TC midspan (see right bottom of Fig. 7), the dipolar / lowfrequency nature of the acoustic emission process is captured well, with waves radiating from the rear cylinder and propagating towards the far field with a wavelength between $30 \mathrm{R}$ to $35 \mathrm{R}$ that is consistent with the expected primary emission frequency. In the $y z$ plane corresponding to a vertical plane cutting through the rear cylinder (see right top

of Fig. 7), the acoustic patterns exhibit a 2-D $\rightarrow$ $3-\mathrm{D}$ evolution. This clearly results from the fact

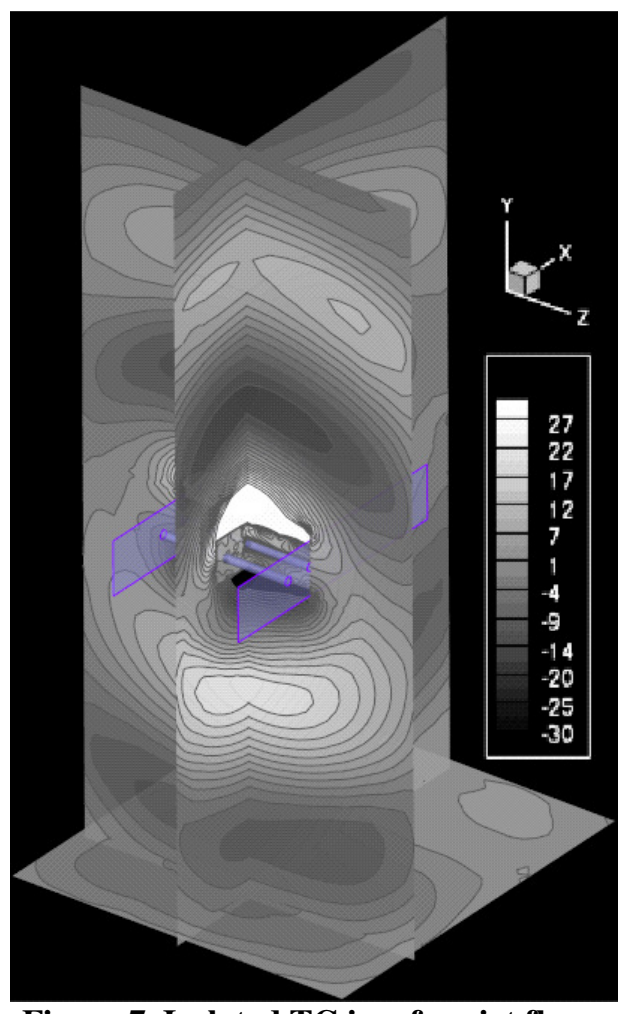

Figure 7. Isolated TC in a free jet flow. Instantaneous perturbation pressure $(\mathrm{Pa})$

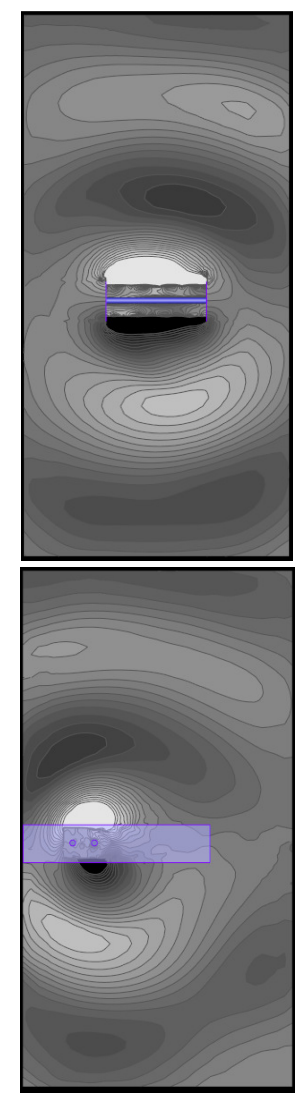


that, after their injection into the CAA computational domain and initial propagation within the bounded region that the cylinders span, the acoustic waves lose the quasi 2-D features inherited from the underlying CFD. These observations were confirmed by the investigation of both the RMS perturbed pressure field and the phase fronts corresponding to a frequency of $f^{\prime}$ prim (not shown here); the dipolar nature of the acoustic radiation was clearly visible, as well as the progression from $2 \mathrm{D} \rightarrow 3 \mathrm{D}$ of the contour patterns; in the near field, acoustic fronts seemed 'flatter' in the $y z$ plane than in $x y$, while in the far field, they all had roughly the same curvature.
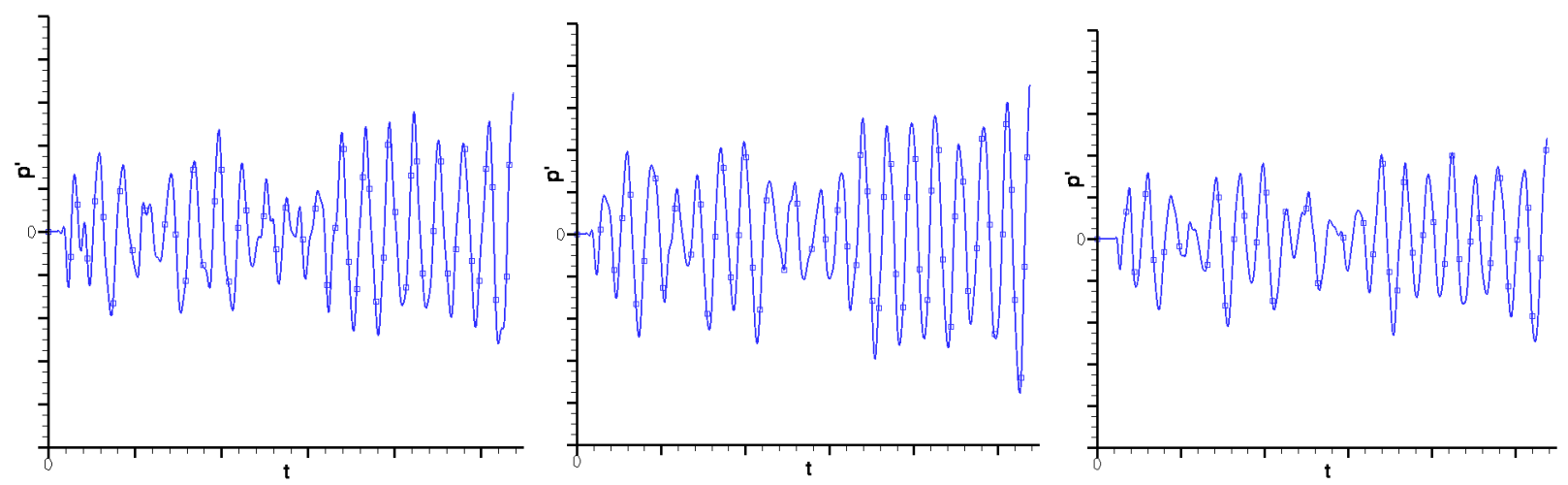

Figure 8. Isolated TC in a free jet flow. Time histories of the perturbation pressure at microphones1 to 3 (from left to right).

Figure 8 presents the time history of the perturbed pressure from the CFD/CAA simulation at the first three microphone locations. Here, one can clearly identify 20 sinusoidal cycles, which correspond to the acoustic forcing delivered by the CFD stage. One can also notice the quite irregular character of the acoustic signals, with amplitudes varying greatly over time. This is in agreement with what had been observed directly in the CFD results and experimentally, corresponding to the intermittent character of aeroacoustic processes characterizing TC flow. One can finally note that, compared to the others, microphone \#3 is characterized by lower acoustic levels. This is obviously explained by the fact that the microphone is positioned further from the source region, which results in more geometric spreading as the signal is propagated. Similar results were obtained for microphones \#4 to \#6.

\section{III.D. 2 Near field comparison and validation}

To assess the accuracy of the CFD/CAA coupled calculation as well as to further validate the surface based coupling technique, the results were compared to the CFD results ${ }^{12,13}$ previously obtained at NASA LaRC.
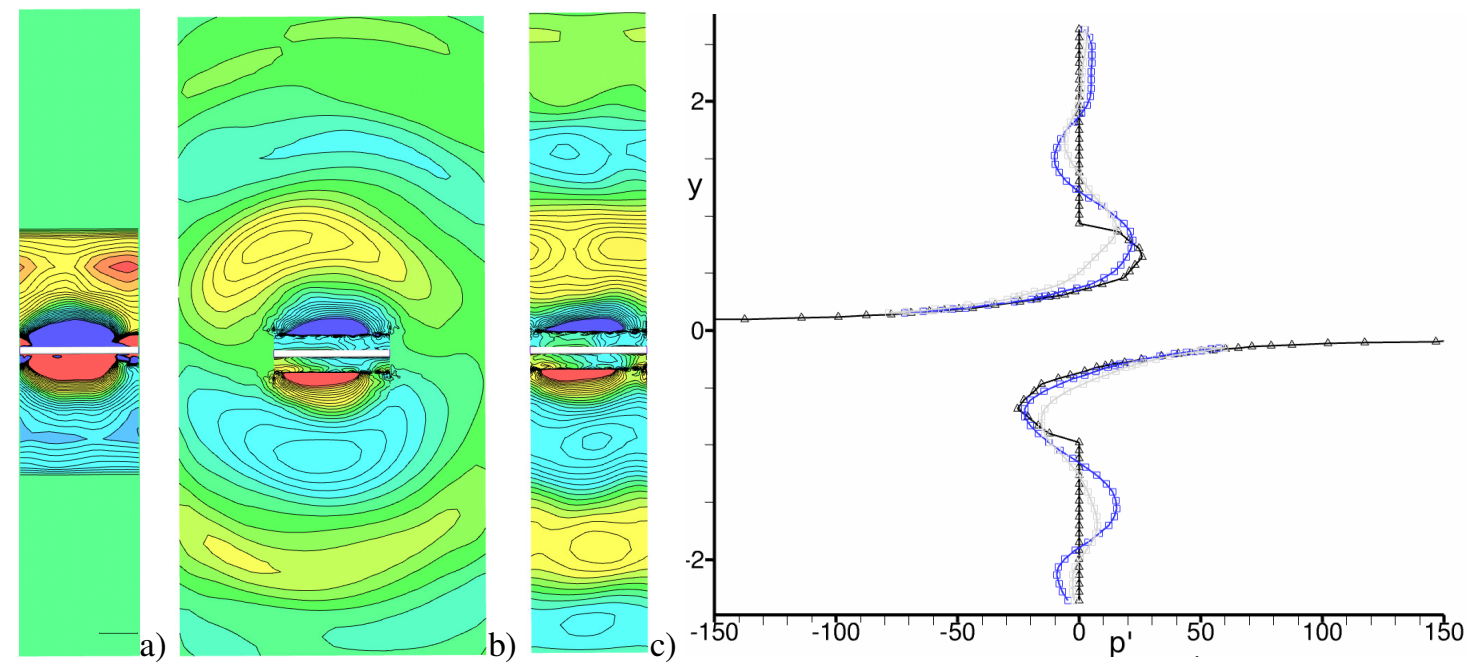

Figure 9. Isolated TC in a free jet flow. Instantaneous perturbation pressure $(t=3 T / 2)$ within the rear cylinder span's yz section (left side) and along its $z=0$ centerline (right side). CFD (left: a, right: black line) vs. CFD/CAA 'periodic' (left: $b$, right: blue line), and 'non periodic' (left: $c$, right: grey line) 
Such a comparison is problematic because both calculations were not exactly representative of the same configuration; indeed, the CFD calculation was run with spanwise periodic boundary conditions producing a nearly 2-D radiation pattern, with waves fronts progressing almost horizontally (see Fig. 9a).However, in the CAA calculation, the source region is embedded in an unbounded region, allowing the acoustic waves to adopt a 3-D propagation behavior, where they rapidly loose their 2-D features initially inherited from the CFD stage (see Fig. 9b). Direct comparison of results led to the observation that, although both amplitudes matched exactly at the surface coupling interface (see right side of Fig. 9), the CFD/CAA signal was weaker than in the CFD counterpart which could partly be explained by the fact that acoustic propagation is characterized by a different spreading rate in 2D $(1 / \sqrt{ } \mathrm{r})$ than in 3D $(1 / \mathrm{r})$. Therefore, an alternative CFD/CAA calculation of the isolated TC configuration was conducted, which aimed at better matching with the CFD numerical set-up; hence, all CAA domains extending beyond the cylinders in the spanwise direction were truncated and lateral periodic boundary conditions applied. This led to CFD/CAA matching better with the CFD, both in terms of the general pattern and amplitude, with a resulting perturbed pressure field characterized by a more spanwise uniform behavior, and acoustic waves with horizontal wave fronts and increased levels (see Fig. 9c). However, as one can see, some discrepancies still remain between both calculations, and their exact origin is still unclear. One possible explanation could be the inability of the CFD stage to accurately account for the near-field prediction of acoustic waves. In particular, although the CFD solver should be capable of accurately propagating such a low-frequency signal relatively accurately over such a short distance, the large wavelength presents a challenge to non-reflecting boundary conditions, and the CFD calculation employed a simple Riemann boundary condition that may have resulted in significant reflections compared with the more advanced boundary conditions used in the CAA code.

Another possibility involves an uncertainty regarding the CFD $\rightarrow$ CAA interpolation process, which - in the present case - had to be conducted not only in time, but also in space. Indeed, according to ongoing theoretical studies being conducted at ONERA $^{19}$, any CFD $\rightarrow$ CAA interpolation process may be subject to various spurious phenomena (spectral aliasing, etc.), which can result in the emergence of errors within the CAA stage. Although all such possible CFD $\rightarrow$ CAA interpolation issues are not completely identified, one can expect that their effects would be minimal for the very low-frequency character of this particular acoustic problem. Whatever the reasons for the discrepancies between the CFD and the CFD/CAA near fields, it is important to remember that CAA methods generally offer higher fidelity/flexibility than CFD in dealing with acoustic phenomena. Consequently, the discrepancies observed here may not indicate any problems with the coupling technique, but instead that the combined CFD/CAA approach may be able to more faithfully simulate configurations of interest. In particular, this suggests that the present CFD/CAA hybrid approach could be used to correct the CFD/FWH results not only from physical artifacts (here the QFF acoustic installation effects), but also from some purely methodological approximations employed for computational simplicity (e.g. periodic boundary conditions that were employed in the CFD simulations).

III.D. 3 Far-field comparison and validation

Because of the difficulty in directly comparing the CFD/CAA near-field to its CFD counterpart, far-field comparisons were made employing the FWH solver used to estimate the isolated TC acoustic signature at microphones \#1 to \#3.
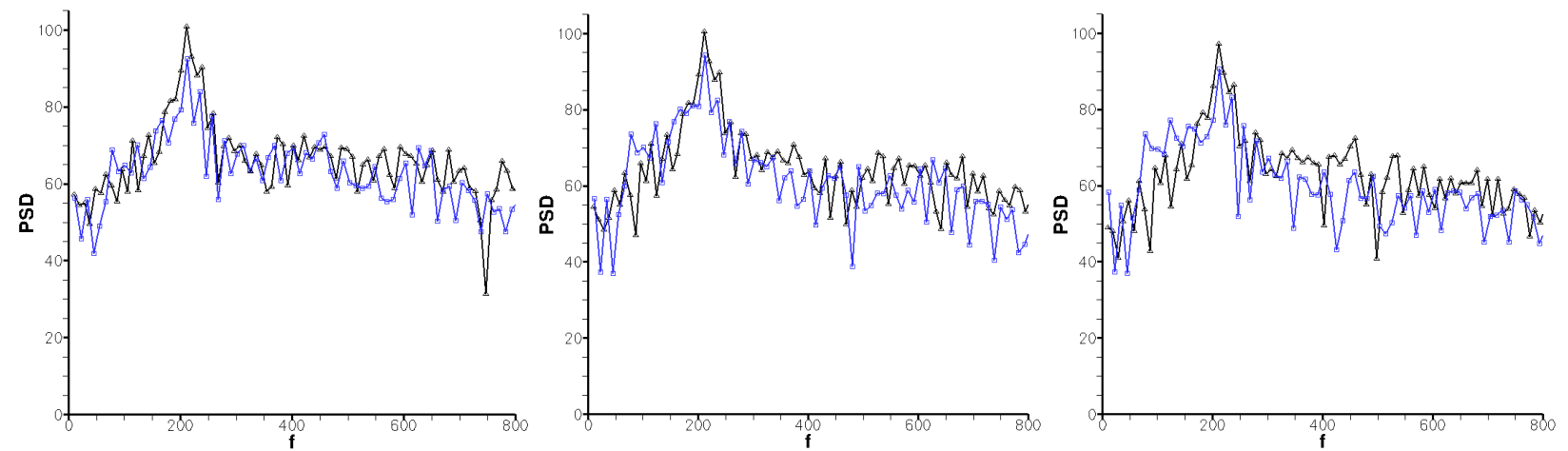

Figure 10. Isolated TC in a free-jet flow. Power Spectral Density of the perturbation pressure propagated/radiated to microphones 1 to 3 (from left to right). CFD/CAA (in blue) vs. CFD/FWH (in black)

The CFD/FWH spectra ${ }^{12}$ were compared to those computed from time history signals sampled directly from the 
CFD/CAA computational domain (see Fig. 8). For each of the first three microphones, Fig. 10 displays the PSD (Power Spectral Density) from both the CFD/FWH and CFD/CAA calculations; although their absolute levels do not match exactly, both spectra have the same trends (over a frequency range extending beyond those well resolved see section II.B.1), and exhibit the same tonal peak at $f=212 \mathrm{~Hz}$ (which corresponds to $f_{\text {prim }}$, to within the Fast Fourier Transform (FFT) bandwidth of $10 \mathrm{~Hz}$ ). Despite the overall agreement in the spectra, they also exhibit some discrepancies, for which various explanations can be proposed. First, for all three microphones, the agreement between the CFD/CAA and CFD/FWH spectra degrades beyond $400 \mathrm{~Hz}$. This can obviously be attributed to the fact that such a $400 \mathrm{~Hz}$ limit corresponds to the highest frequency for which a good resolution was ensured by the CAA grid. Second, considering the three plots successively reveals that the divergence between the CFD/FWH and CFD/CAA spectra seems to be more pronounced for microphone \#3, located downstream of the cylinders. This could be due to spurious effects from the coupling interface caused by the passage of hydrodynamic events convected by the wake. Indeed, in the present case, the function used to artificially confine the forcing to the vicinity of the cylinders could have been insufficient to prevent all (intermittent and relatively strong) hydrodynamic events from corrupting the downstream section of the coupling interface. Furthermore, the CFD/FWH and the CFD/CAA spectra exhibit an amplitude mismatch at the tonal frequency. Such an amplitude mismatch cannot be associated with the inability of the CFD stage to accurately account for the near-field acoustic propagation since, in the present case, only the solid surface data from the CFD was used in the FWH calculation. In the same way, such amplitude discrepancies cannot be attributed to the inability of this $\mathrm{FWH} /$ solid surface computation to account for possible quadrupole noise sources, since alternative $\mathrm{FWH}$ /porous surface calculations have been conducted that produced no real difference in the far-field results. However, a possible reason for the mismatch in amplitude could be because the periodic boundary conditions in the CFD calculation imposed perfect spanwise correlation at both cylinders' extremities. Consequently, the FWH extrapolation could have produced a non-negligible over-estimation of acoustic levels in the mid-span plane, precisely where the microphones are located. Finally, the differences in amplitude levels could also have been associated with the FWH-related FFT post-processes.

Therefore, in an effort to make the procedures for calculating the far-field noise as close as possible between the two simulations, the CFD/CAA data was used as input to the FWH solver and compared with the CFD/FWH computed signals.
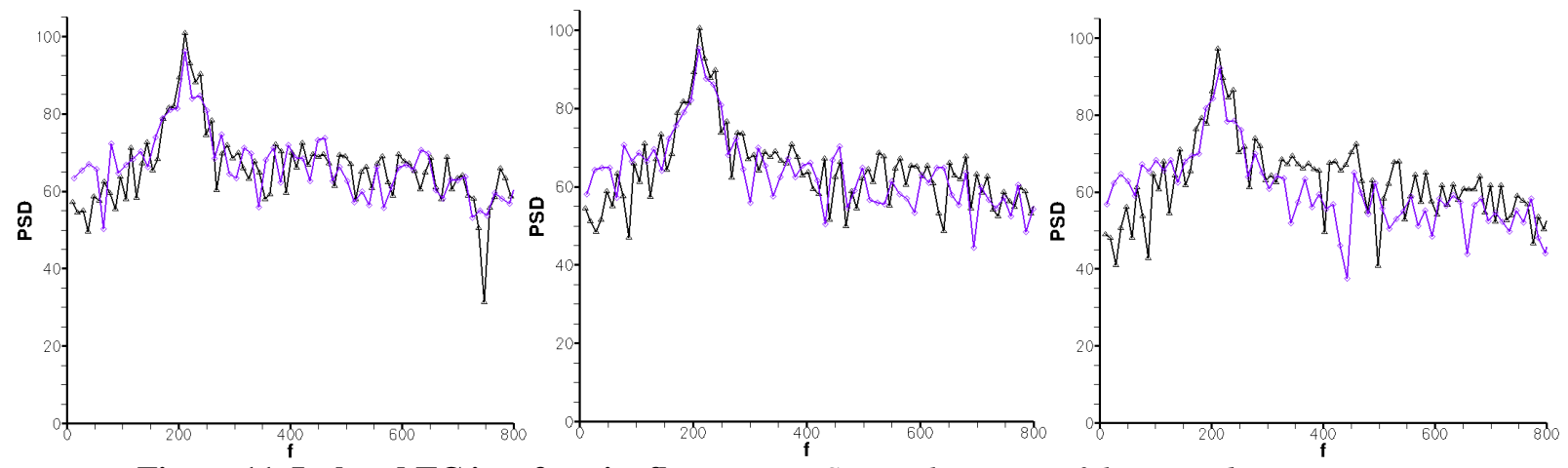

Figure 11. Isolated TC in a free-jet flow. Power Spectral Density of the perturbation pressure propagated/radiated to microphones 1 to 3 (from left to right). CFD/CAA/FWH (in purple) vs. CFD/FWH (in black)

As one can see in Fig. 11, the consistency between these results is much better in terms of both amplitude and trends. Although we have not included the experimental data in these figures, the comparisons of the CFD/FWH results with the experimental data in Ref. 12 are quite close, and the differences are often within the variability observed in the experiment. Hence, we can conclude that the current CFD/CAA/FWH results are reproducing the experimental observations with sufficient accuracy to assess the installation effects in the QFF. The mismatches previously observed when comparing the CFD/CAA and CFD/FWH results are from the differences in how the farfield noise is estimated. Furthermore, both the hybrid methodology and the underlying surface coupling technique must be functioning as intended. From a more global point of view, such an observation nevertheless underscores the difficulty of comparing calculation results coming not only from different methods, but also from different combinations of methods. Indeed, it is not only the respective accuracy / validity of all involved methods that matters, but also their linking. 


\section{E. Installed TC in a Free-Jet Flow}

To isolate the reflection / diffraction effects produced by the QFF experimental setup on the TC acoustic signature, the previous isolated configuration was modified to include the main QFF elements (side plates, collector plate, nozzle), with the steady mean flow still being considered as a free jet (see left side of Fig. 4).

\section{III.E.1 Calculation results and early analysis}

Views of the instantaneous perturbation pressure field are presented in Fig. 12, at a time 2/3rd of the way through the calculation. A comparison between these results and those from the isolated TC configuration (see Fig. 7) reveals that the QFF devices modify the acoustic field in several ways. First, between the side plates, acoustic waves exhibit an enhanced 2-D character, as well as increased amplitudes. This can be attributed to the partial confinement that acoustic waves experience. Indeed, once generated, acoustic waves are trapped on two sides by the side plates, which then act on them as a wave guide. Secondly, outside of the side plates, acoustic waves display an enhanced 3-D character, while they exhibit higher amplitudes between the plates (especially in the mid-span $y z$ plane, compare right/top views of Figs. 7 and 12). The change from $2 \mathrm{D} \rightarrow 3 \mathrm{D}$ in the propagation behavior occurs when acoustic waves are diffracted by the side plates' edges, which 'break up' the initial 2-D character of emitted waves, promoting a 3-D / free space propagation behavior. Finally, further away from the side plates, acoustic waves maintain their 3-D nature, with either slightly higher or lower amplitudes, depending on the location. As will be shown, such relative gains / losses in amplitude seem to result primarily from the cumulative effect of all QFF components, which cause constructive / destructive interference on the overall acoustic field. These various acoustic installation effects appear even more explicitly when comparing the present acoustic phase field to its isolated TC counterpart (see Fig. 13). First, one can notice the higher curvature that acoustic wave fronts adopt in the $y z$ plane, which reflects the transition from 2-D $\rightarrow 3-D$ propagation that the side plates enhance. Furthermore, a direct comparison of other views reveals how both the collector plate and the nozzle modify the acoustic patterns in their immediate vicinity, as revealed by the strong discontinuities characterizing the acoustic phase field (and thus wave fronts) nearby these obstacles. These local

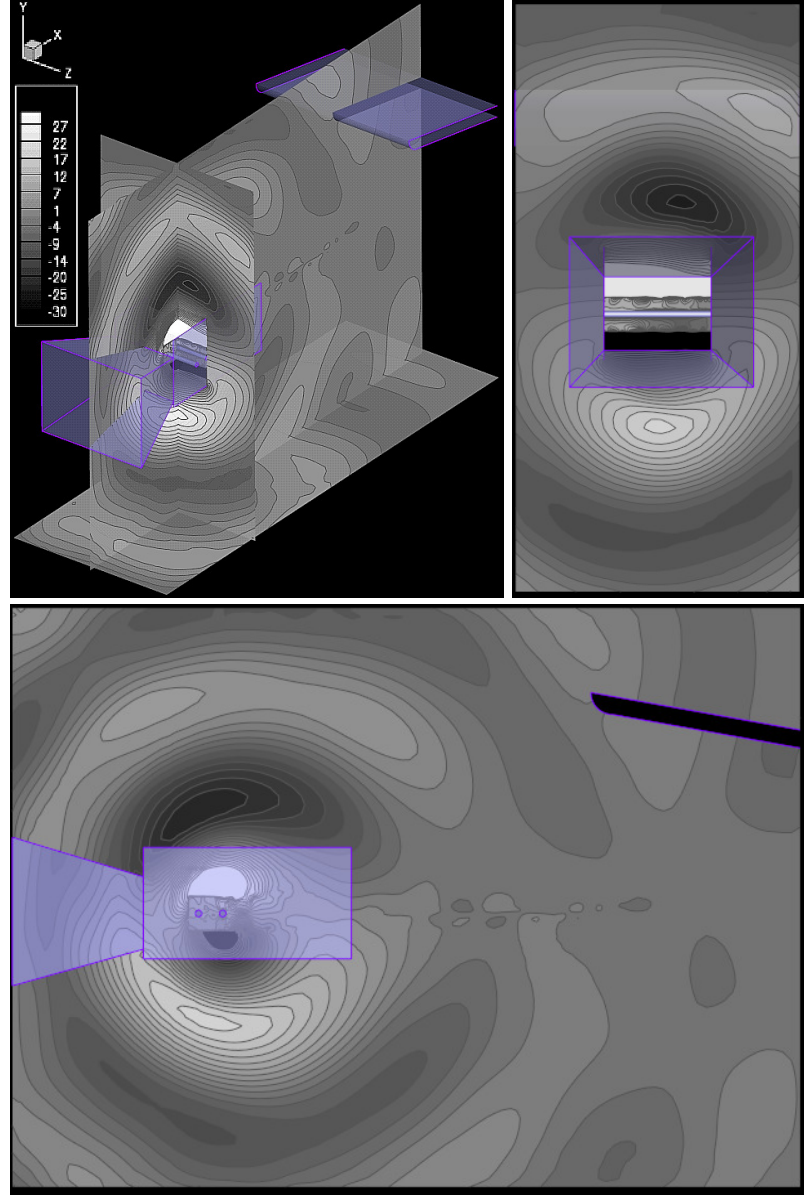

Figure 12. Installed TC in a free jet flow.

Instantaneous perturbation pressure $(\mathrm{Pa})$ modifications of the acoustic phase by the solid obstacles indirectly indicate the reflection / diffraction phenomena, which can have a more global impact due to the backscatter of acoustic waves into the domain. Cumulatively, all these effects might explain the amplitude changes that the acoustic field exhibits throughout the domain, as indicated by the comparison of both RMS perturbed pressure fields (not shown here).

\section{III.E.2 Further assessment of acoustic installation effects by QFF components}

To more clearly identify the origin of all various acoustic installation effects occurring here, three RMS 'delta' maps were produced, which are presented in Fig. 14. These maps were calculated from the RMS perturbed pressure fields associated with each of the various 'installed TC' configurations successively computed. The configuration without any QFF components was taken as the baseline, and the sequence of simulations highlights the differences in RMS levels in the acoustic field when the QFF components were progressively installed. Consequently, these delta maps indicate the respective contribution of each QFF component (side plates, collector plate and nozzle, successively) to the overall acoustic installation effects; the left side of Fig. 14 confirms that the side plates primarily contribute to a 

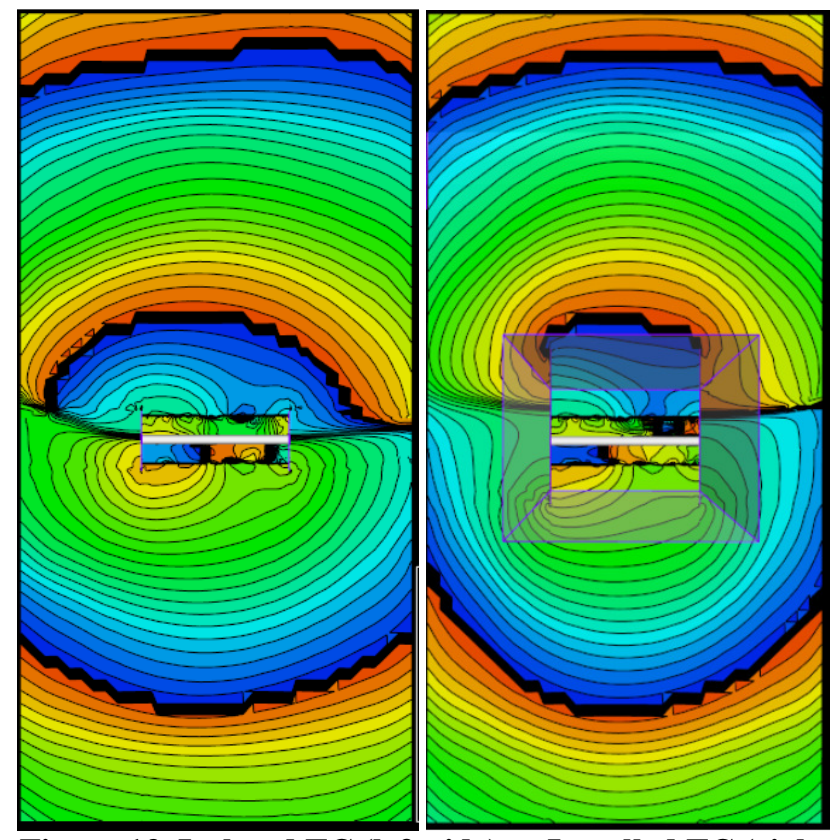

Figure 13. Isolated TC (left side) vs Installed TC (right side) in a free-jet flow. Acoustic phase field within the $y z$

plane, drawn over 12 iso-levels ranging from $-\pi$ to $\pi$ (relatively important) increase in acoustic levels between them and in their immediate vicinity. Once again, this appears to be related to the partial confinement that the plates impose on the acoustic waves, resulting in a non-negligible increase in amplitude. As an example, most of the strongly reinforced levels $(+6.5 d B)$ occur between the side plates, downstream the TC (see left/top of Fig. 14). The figure also exhibits a lack of $x y$ or $y z$ symmetry (see left/top and left/bottom of Fig. 14, respectively), which results from the asymmetry in both the acoustic source and the position of the side plates relative to the cylinders. The side plates do not always lead to a reinforcement of acoustic levels. Indeed, shadow zones (in blue) are evident in the left/bottom of Fig. 14 caused by the shielding effect of the plates. Finally, the side plates have less of an impact on the acoustic field radiated further into the domain, with amplitude modifications of less than $2 d B$. In particular, the effect they have on the acoustic levels recorded at the microphones never exceeds $1.7 d B$. The effect of the collector plate (relative to the just the side plates) is highlighted by comparing the center view of Fig. 14 to its left counterpart. The primary effect seems to be slight changes in the local acoustic levels all over the domain. As previously stated, the reflecting / diffracting waves off the collector plate probably induce constructive and destructive interference throughout the whole domain.

Finally, the additional effect of the nozzle can be inferred by comparing the right view of Fig. 14 to its center counterpart. Similarly to the collector plate, the nozzle acts as a reflecting / diffracting surface which results in slight alterations of acoustic levels all over the domain. However, the current observations must be considered a worst case scenario, because the nozzle was considered as rigid, whereas in the experiments, it was acoustically treated with absorbing material (foam).

Despite some relatively strong modifications to the propagating acoustics between the side plates, only relatively small changes are observed where the microphones are located (see right/top view of Fig. 14). More precisely, as detailed on Table 1 , the overall delta effect induced by all QFF components on the acoustic levels recorded at a given microphone rarely exceeds $3 d B$ (i.e. $40 \%$, in linear amplitude). In particular, the

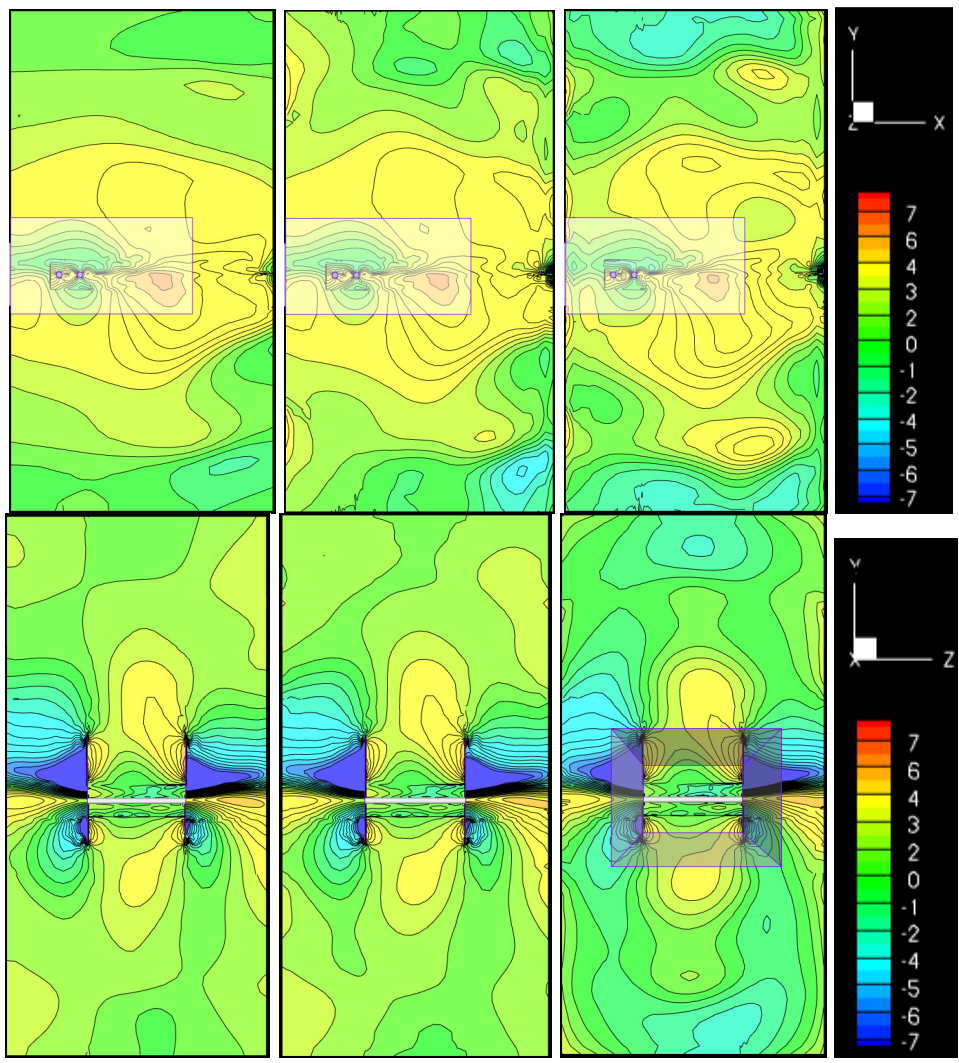

Figure 14. Installed TC within a free jet flow. Deltas of the RMS perturbed pressure (as PSD in $d B$ ) in both the xy plane (top) and the $y z$ plane (bottom). Delta between the baseline, open configuration and calculations with the side plates (left), the side and collector plates (center) and all the elements (right) 
microphones located just downstream of the cylinders (mics \#2 and \#5) are the ones for which the overall acoustic installation effects are the weakest. For some microphones, adding a particular QFF component may mitigate the effect induced by others devices (for example, the effect of the nozzle on mics \#1, \#4 and \#5). Furthermore, the lower microphones (mics \#4 to \#6) are subjected to similar changes in the acoustic levels as the upper ones (mics \#1 to \#3), although they are located further away from the collector plate. These two observations indicate that each microphone is subject to a global cumulative influence of all QFF components, rather than to a local effect induced by the closest QFF component. In other words, the acoustic installation effects appear to be driven by the constructive and destructive interference from the cumulative reflection / diffraction off of all the QFF components. In addition, both the observer's location and the acoustic source dynamics play an important role. This behavior is to be expected because of the very low-frequency character of the noise source, make it relatively non-compact with respect to the experimental set up. From an acoustic installation point of view, the various QFF devices interact quite closely, and cannot really be considered separately.

Table 1. Installed TC within a free jet flow. Deltas in the PSD $(d B)$ caused by the QFF components on the RMS perturbation pressure field radiated to the microphones

\begin{tabular}{|c|c|c|c|c|c|c|}
\hline Delta effect $(\boldsymbol{d B})$ & Mic \#1 & Mic \#2 & Mic \#3 & Mic \#4 & Mic \#5 & Mic \#6 \\
\hline Side plates & 1.34 & 0.58 & 1.68 & 1.15 & 0.72 & 1.59 \\
\hline Side plates + Collector plate & 3.4 & -0.15 & 1.78 & 3.19 & 0.28 & 2.32 \\
\hline Side plates + Collector plate + Nozzle & $\mathbf{2 . 8 2}$ & $\mathbf{- 1 . 3 6}$ & $\mathbf{2 . 2 4}$ & $\mathbf{2 . 7 6}$ & $\mathbf{0 . 2 3}$ & $\mathbf{3 . 4 4}$ \\
\hline
\end{tabular}

The overall effects previously highlighted are confirmed by Fig. 15, which displays the time histories of perturbed pressure signals recorded at the microphone locations for the fully installed TC, and compares them to the ones obtained for the isolated TC (cf. Fig. 8).
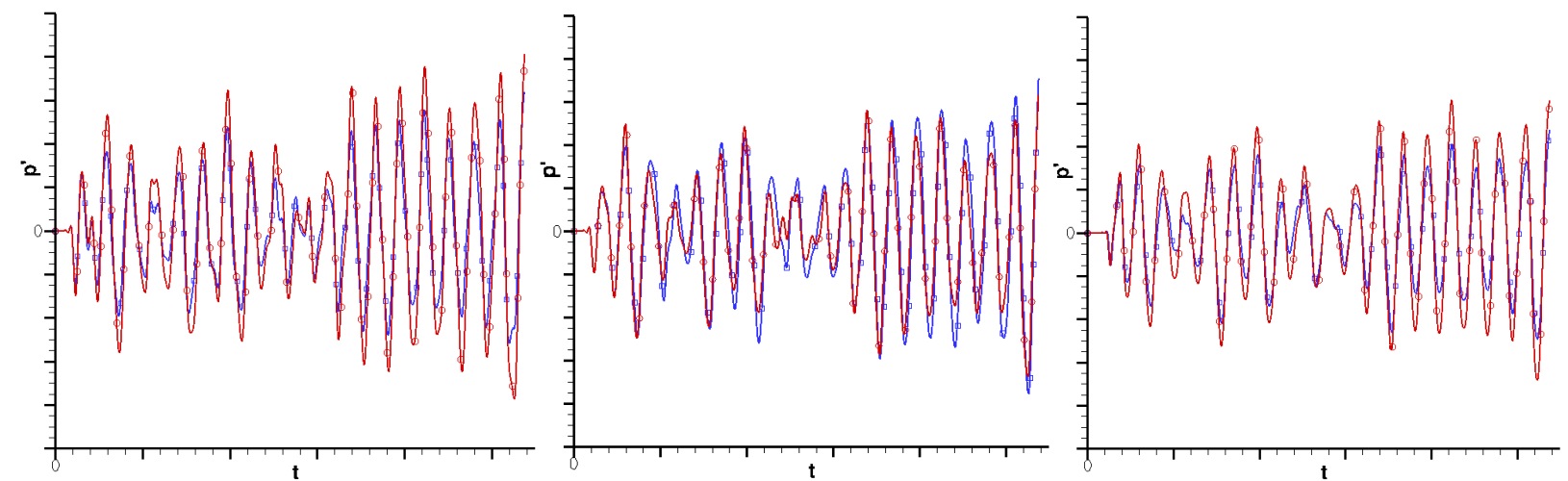

Figure 15. Installed TC in a free jet flow. Time histories of the perturbation pressure at microphones 1 to 3 (from left to right). Installed (in red) vs. isolated (in blue) configurations

For all six microphones (only three of which were shown here), the oscillatory behavior of the acoustic signatures is similar, but the modulated amplitude is different. The change in the effective amplitude never exceeds $40 \%$, with modifications varying between $-1.35 \mathrm{~dB}$ and $+3.45 \mathrm{~dB}$, depending on the microphone location. More precisely, compared to the isolated configuration, the microphones that are located upstream (mic \#1 and \#4) and downstream (mic \#3 and \#6) see a systematic increase in acoustic levels. In contrast, the two microphones located just downstream of the cylinders (mic \#2 and \#5) are characterized by acoustic levels that vary between being stronger and weaker than the baseline, but with an overall decrease from the baseline.

Figure 16 displays the PSD (Power Spectral Density) spectra associated with the time history signals of Fig. 15. The trends previously observed in the time domain are also exhibited here, with all fully-installed TC spectra matching the isolated case very closely, not only in terms of amplitude, but also in terms of shape. 

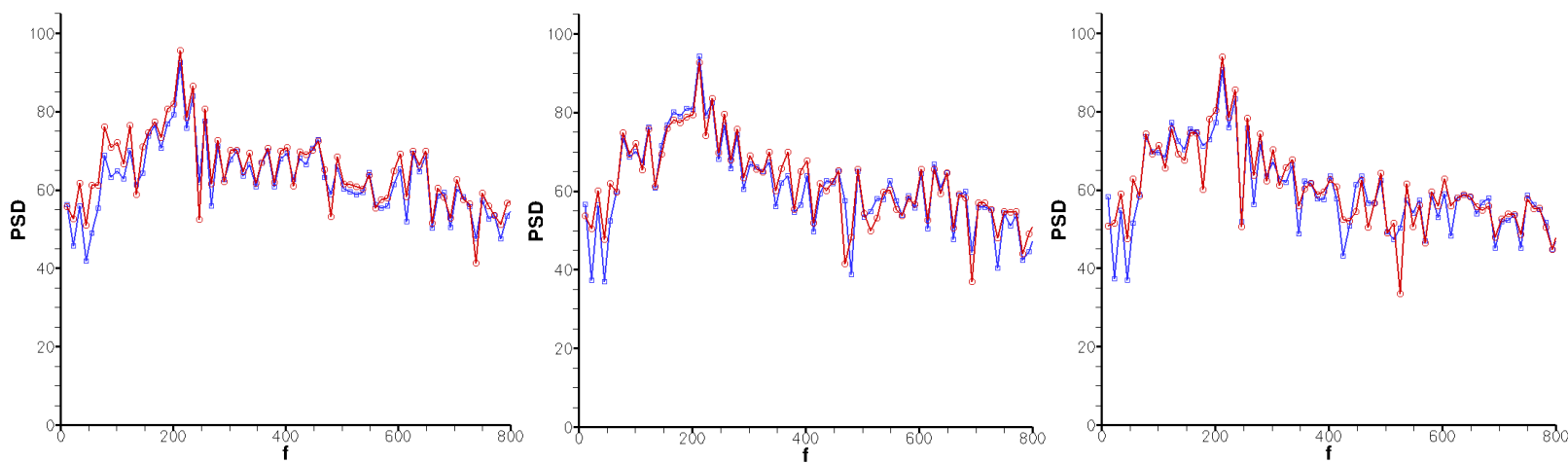

Figure 16. Installed TC in a free jet flow. Power Spectral Density (in $d B$ ) of the perturbation pressure at microphones 1 to 3 (from left to right). Installed (in red) vs. isolated (in blue) configurations

\section{III.E.3 Preliminary conclusions}

Based on the current observations, the overall reflection / diffraction effects produced by the QFF components on the TC acoustic signature are not prominent. In particular, at the microphone locations where the NASA/LaRC experimental data were acquired, such effects only lead to amplitude differences that do not exceed $40 \%$. These effects are mainly due to the acoustic reflection / diffraction by the large-scale facility features (nozzle, side plates, collector), which are found to make comparable contributions to the overall effect of facility installation. First, it has to be pointed out that these conclusions are valid only for the configuration that was assessed here, and should not be generalized to other QFF experiments, especially those that involved noise sources that differ greatly from lowfrequency, dipole one investigated here. Furthermore, the current conclusions and even the quantitative numbers are consistent with those provided by NASA/LARC researchers in their earlier assessment of QFF installation effects, achieved using an Equivalent Source Method ( $F S C$ solver) forced by equivalent dipoles ${ }^{14}$. From a methodological point of view, this tends to indicate that the present TC configuration seems to be adequately modeled by simple equivalent sources. On another hand, this also indicates that the current CFD/CAA hybrid methodology seems to be as appropriate for evaluating installation effects as other (and sometimes simpler) computational approaches, while allowing the possibility of including further realism (source description, complete acoustic installation effects, refraction effects, etc.)

\section{F. Installed TC, Within the QFF Confined / Sheared Jet Flow}

To assess the convection and refraction effects of the QFF jet mean flow on the TC acoustic signature, the previous installed TC configuration was used in conjunction with the confined / sheared jet flow (see Fig. 4).

\section{III.F.1 Calculation results and preliminary analysis}

Figure 17 shows the instantaneous perturbed pressure field, at a time $2 / 3 \mathrm{rd}$ of the way through the calculation. By comparing these results to those from the calculation without jet spreading (see Fig. 12), one can notice the alterations that such a realistic jet flow makes on the acoustic field. The acoustic patterns are modified in the $x y$ plane, but they remain roughly the same in the $y z$ plane. The modifications are obviously related to both the (partial) convection and refraction effects characterizing such a confined / sheared jet flow. Indeed, in contrast to the previous cases, for which the whole domain was subject to convective effects, the present medium is free of

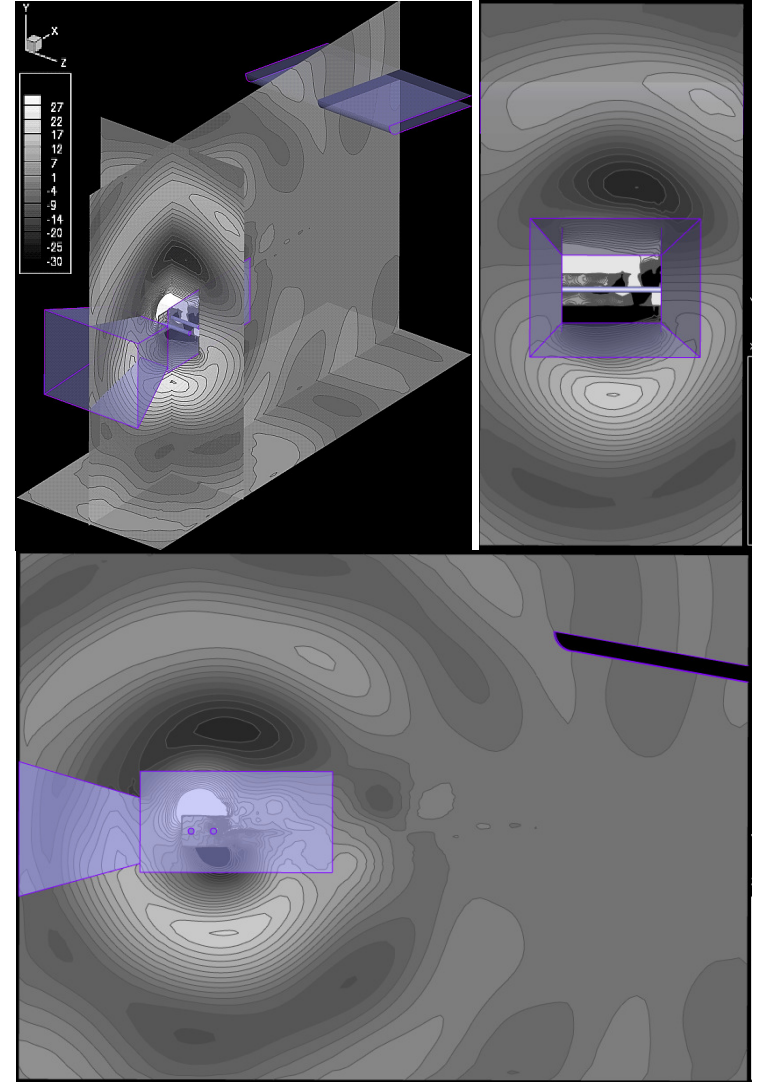

Figure 17. Installed TC within the QFF confined / sheared jet flow. Instantaneous perturbation pressure $(\mathrm{Pa})$ 
any convection, except within the limited area where the jet exists (i.e. between the side plates and in the wake). Furthermore, this more realistic mean flow has strong gradients in the shear layers surrounding the jet, so it is more likely to refract acoustic waves. However, such refraction effects are expected to have little impact on the present TC acoustic emission, which is characterized by a very low frequency (and thus a long wavelength, with respect to the shear layer thickness). In particular, a closer examination of Fig. 17 tends to indicate that the lack of convection outside of the jet leads to most of the modifications to the acoustic field. Indeed, by comparing the center views of Figs. 12 and 17, one can notice that acoustic waves in the current calculation exhibit both a lower amplitude and a longer wavelength as they propagate in the upstream direction, whereas the opposite is true in the downstream direction. This behavior is consistent with convection effects, which generally lead to higher amplitudes and shorter wavelengths for signals propagating upstream.

\section{III.F.2 Further assessment of acoustic installation effects by QFF jet flow}

The modification of the acoustic field by the (restricted) convective effects characterizing the present confined / sheared jet flow appears more clearly in Fig. 18, which presents the acoustic phase field associated with the primary emission frequency $\left(f=f^{\prime}\right.$ prim $)$, and compares it to its unsheared counterpart.
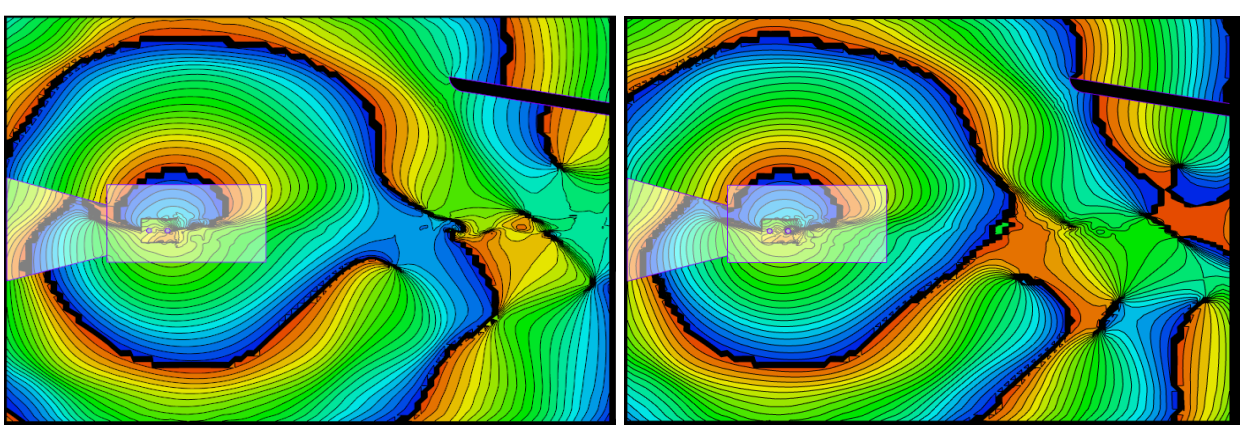

Figure 18. Installed TC within both a free (left side) and the QFF confined / sheared (right side) jet flows. Acoustic phase $\left(f=f^{\prime}\right.$ prim $)$ field, drawn over 12 iso-levels ranking from $-\pi$ to $\pi$

Here, one can see how, outside the jet, acoustic waves are no longer slowed when propagating upstream (compare the wave fronts inside the nozzle to those outside), nor sped up when progressing downstream (see what occurs near the collector). This simply results from the fact that, depending on the flow considered, acoustic wave fronts are more or less compressed / stretched along the $\mathrm{x}$-axis.

The RMS of the perturbation pressure field associated with the present configuration (not shown here) also revealed that the levels appear to be almost identical to those that had been previously obtained for a free jet flow. Such an observation is not inconsistent with the remarks that were previously made regarding the way acoustic patterns are modified by the (partial) convection effects. The convection primarily affects the phasing of the signals, and, once RMS-averaged, all the influence of acoustic phase shifts simply vanish. Furthermore, any local amplitude gain / loss caused by convection effects are negligible since the Mach number is low $(M=0.128)$ and the wavelength is long $(\lambda=1.9 \mathrm{~m}=6.3 \mathrm{ft})$.

These conclusions are confirmed by Fig. 19 (left side), which displays part of the time history of the perturbation pressure recorded at the first three microphone locations, superimposing them with the signals already obtained for two previous cases. Compared to the isolated and fully-installed configurations (both combined with the same freejet mean flow), the acoustic signals from the present calculation are slightly out of phase. However, their amplitude roughly equals those from the installed/free-jet flow configuration. When looking more closely at these results, it appears that the tendencies are consistent with what was expected; acoustic signals reach the upstream microphones (\#1 and \#4) slightly sooner than previously - because they are no longer slowed down by convection effects. Conversely, they now reach the downstream microphones (\#3 and \#6) slightly later than previously - which can be explained by the fact that they are no longer carried along by the flow. And, finally, acoustic signals reach the microphones located above and below the cylinders (\#2 and \#3) without modification - this being simply due to the fact that, previously, no convective effect was acting in that direction, which is orthogonal to the flow. As expected, once Fourier analyzed, these time history signals deliver spectra that closely match the ones that had been previously obtained for the installed / free-jet flow configuration (see Fig. 19, right side). This further confirms the very slight impact that such a realistic sheared/confined jet flow has on the acoustic field characterizing the fully-installed TC configuration. Again, such a conclusion must be tempered by the fact that, here, the acoustic emission was of very low frequency, which rendered it almost insensitive to diffraction effects as waves propagated across the jet shear layers. Such a situation is quite unusual because the small scale of experimental models leads to noise emission at very high frequency (50 $\mathrm{kHz}$ is not rare). 

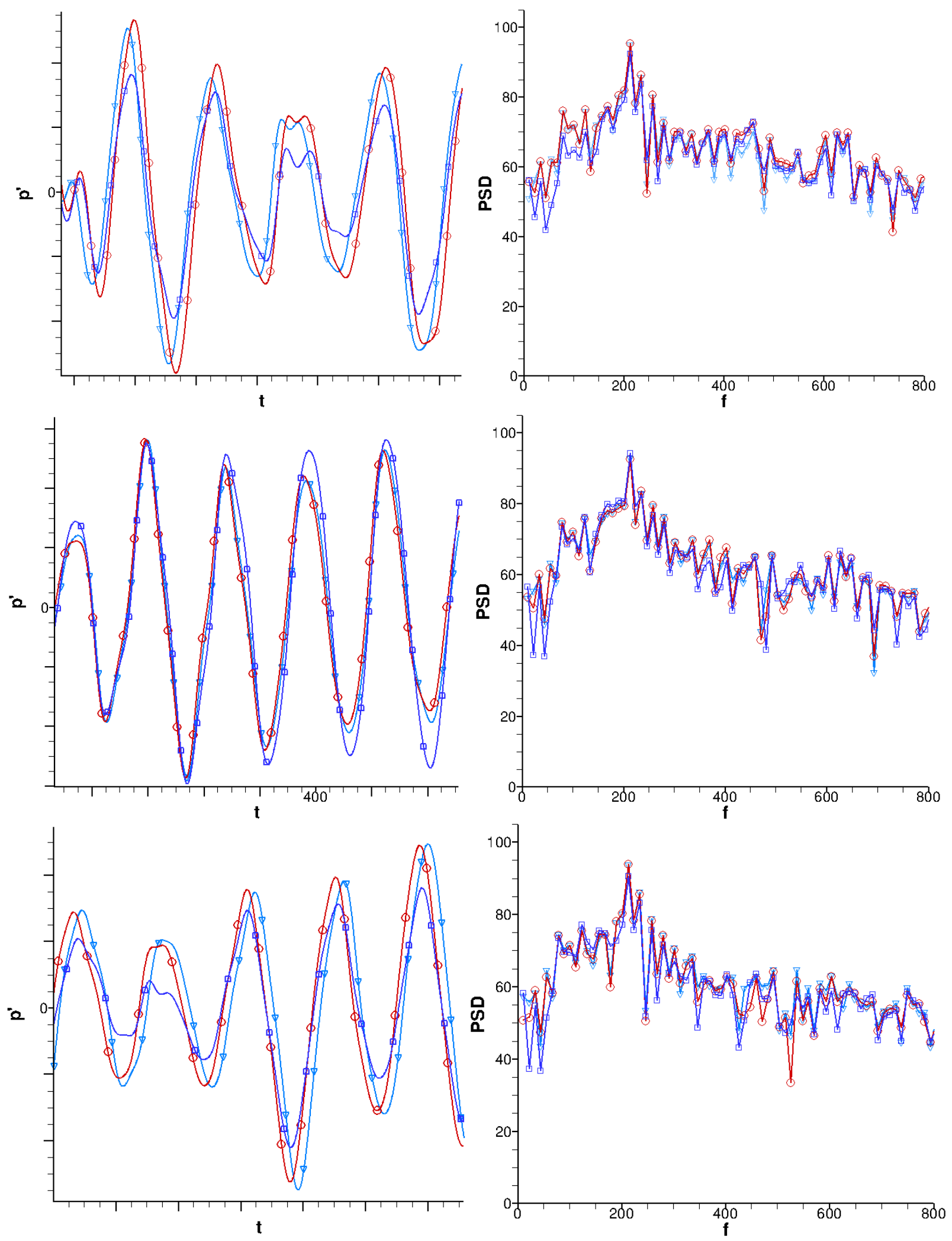

Figure 19. Installed TC in the QFF. Partial time history of the perturbation pressure at mics 1 to 3 (from top to bottom on left side). PSD (in dB) of the perturbation pressure at mics 1 to 3 (from top to bottom on right side). Installed TC / QFF flow (light blue) vs. installed TC / free- jet flow (red) and isolated TC / free-jet flow (blue) 


\section{Conclusions and Perspectives}

The present study described a numerical investigation of the acoustic installation effects related to the Tandem Cylinder aeroacoustic experiments in the NASA/LaRC QFF facility. The numerical computations were conducted using a hybrid approach involving a weak coupling between the nearfield CFD computation and the CAA calculation aimed at propagating the nearfield information to the far field. The computations employed the so-called 'surface' coupling approach, which was recently improved to better handle acoustic backscattering that is typical of airframe configurations installed within a wind tunnel facility. Several CFD/CAA coupled calculations of the TC configuration were conducted, all of which involved the combination of 1) a common CFD stage that utilized previously obtained numerical solutions, and 2) various CAA stages focused on different aspects of the installation effects. Specifically, the latter allowed numerical evaluations of the effect of each component (e.g., nozzle, collector, etc.) or a feature characterizing the NASA/LaRC QFF facility (e.g., confined jet vs. co-flow). The impact of each installation effect on the acoustic signature of the TC configuration was assessed. The primary conclusion from these computations is that the total magnitude of the installation effects associated with the TC model in the QFF is rather modest. In particular, at the 6 microphone locations used during testing, the installation effects are expected to account for amplitude differences of less than $40 \%$ in magnitude (with the change in amplitude varying between $-1.35 \mathrm{~dB}$ and $+3.45 \mathrm{~dB}$, depending on the microphone location). Most of these effects are due to the QFF components themselves, resulting either from the confinement of the acoustic emission by side plates, or from additional reflection / diffraction of radiated waves by both the collector plate and the nozzle. Very slight modifications are due to the QFF jet flow, which mainly results from convection being restricted to the region of the jet. From a methodological point of view, the present results contribute to a more meaningful comparison between QFF acoustic data and noise predictions based on the free-field TC configuration. Such results are thus an important step in employing the QFF measurements in semi-empirical prediction techniques for landing gear noise, such as that proposed by Lopes et al. ${ }^{20}$. Furthermore, these results also provide an additional validation / application of the CFD/CAA surface coupling, and pave the way for more systematic utilization of such techniques for solving more complex problems involving significant acoustic installation effects, for which commonly used computational approaches (such a CFD/FWH one) are not applicable ${ }^{21}$.

Obviously, the conclusions drawn in this study are valid only for the configuration that was assessed here, and should not be generalized to other QFF experiments, especially those involving noise sources that differ greatly from the low-frequency, dipole dominating the noise radiation associated with the TC configuration. In particular, considering the relatively high frequencies characterizing usual airframe noise sources, most QFF acoustic experiments should be more strongly influenced by the diffraction across the shear layers surrounding the QFF jet flow. For this reason, follow-on computations are planned to extend the present results to include high frequency sources such as simple distributions of acoustic monopoles and dipoles to provide a more complete numerical assessment of the diffraction effects associated with the spreading of the QFF jet. Several jet flow speeds and spreading angles will be studied, including a wide range of frequencies. This will provide an opportunity to check the validity of the infinitely thin shear layer correction that is commonly applied to QFF experimental data. The outcome of this prospective study will help assess further acoustic installation effects that may be important in the type of testing typically done in the NASA/LARC QFF facility.

\section{Acknowledgments}

The present work was funded by ONERA and conducted while the first author was in residence at NASA Langley Research Center as part of a NASA-ONERA collaboration related to airframe noise. The authors would like to thank Dr. Florence Hutcheson and Mr. Daniel Stead for the detailed information regarding the QFF experiment. The authors would also like to acknowledge Dr. Ana Tinetti, whose past work and informal exchanges helped guide the present study.

\section{References}

${ }^{1}$ Polacsek, C., Burguburu, S., Redonnet, S. and Terracol, M., "Numerical Simulations of Fan Interaction Noise using a Hybrid Approach," AIAA Journal, Vol. 44, No. 6, June 2006.

${ }^{2}$ Ffowcs Williams, J. E. and Hawkings, D. L., "Sound Generation by Turbulence and Surfaces in Arbitrary Motion," Philosophical Transactions of the Royal Society of London A, Vol. 342, 1969, pp. 264-321.

${ }^{3}$ Redonnet, S., "On the Numerical Prediction of Aerodynamic Noise via a Hybrid Approach - Part 1: CFD/CAA Surfacic Coupling Methodology, Revisited for the Prediction of Installed Airframe Noise Problem," AIAA Paper 2010-3709, 16th AIAA/CEAS Aeroacoustics Conference, Stockholm, Sweden, June 2010.

${ }^{4}$ Redonnet, S., "Simulation de la Propagation Acoustique en Présence d'écoulements Quelconques et de Structures Solides, par Résolution Numérique des équations d'Euler," PhD Thesis, Université Bordeaux I, 2001. 
${ }^{5}$ Manoha, E., Herrero, C., Sagaut, P. and Redonnet, S., "Numerical Prediction of Airfoil Aerodynamic Noise," AIAA Paper 2002-2573, 8th CEAS/AIAA Aeroacoustics Conference, Breckenridge, USA, June 2002.

${ }^{6}$ Guenanff, R., "Couplage Instationnaire Navier-Stokes/Euler pour la Génération et le Rayonnement des Sources de Bruit Aérodynamique," PhD Thesis, Rennes University, 2004, No. 3138.

${ }^{7}$ Terracol, M., Manoha, E., Herrero, C., Labourasse, E., Redonnet, S. and Sagaut, P., "Hybrid Methods for Airframe Noise Numerical Prediction," Theoretical and Computational Fluid Dynamics, Vol. 19, No.3, July 2005.

${ }^{8}$ Jenkins, L. N., Khorrami, M. R., Choudhari, M. M. and McGinley, C. B., "Characterization of Unsteady Flow Structures Around Tandem Cylinders for Component Interaction Studies in Airframe Noise," AIAA Paper 2005-2812, 11th AIAA/CEAS Aeroacoustics Conference, Monterey, USA, May 23-25, 2005.

${ }^{9}$ Jenkins, L. N., Neuhart, D. H., McGinley, C. B., Choudhari, M. M. and Khorrami, M. R., "Measurements of Unsteady Wake Interference Between Tandem Cylinders," AIAA Paper 2006-3202, 36th AIAA Fluid Dynamics Conference and Exhibit, San Francisco, USA, June 5-8, 2006.

${ }^{10}$ Hutcheson, F. V. and Brooks, T. F., "Noise Radiation from Single and Multiple Rod Configurations," AIAA Paper 20062629, 12th AIAA/CEAS Aeroacoustics Conference, Cambridge, USA, May 2006.

${ }^{11}$ Neuhart, D. H., Jenkins, L. N., Choudhari, M. M. and Khorrami, M. R., "Measurements of the Flowfield Interaction Between Tandem Cylinders," AIAA Paper 2009-3275, 15th AIAA/CEAS Aeroacoustics Conference, Miami, USA, May 2009.

${ }^{12}$ Lockard, D. P., Khorrami, M. R., Choudhari, M. M., Hutcheson, F. V. and Brooks, T. F., "Tandem Cylinder Noise Prediction," AIAA Paper 2007-3450, 13th AIAA/CEAS Aeroacoustics Conference, Roma, Italy, May 2007.

${ }^{13}$ Lockard, D. P., Choudhari, M. M., Khorrami, M. R., Neuhart, D. H., Hutcheson, F. V. and Brooks, T. F., "Aeroacosutic Simulations of Tandem Cylinders with Subcritical Spacing," AIAA Paper 2008-2862, 14th AIAA/CEAS AeroAcoustics Conference, Vancouver, Canada, May 2008.

${ }^{14}$ Tinetti, A. F. and Dunn, M. H., "Acoustic Simulations of an Installed Tandem Cylinder Configuration," AIAA Paper 20093158, 15th AIAA/CEAS AeroAcoustics Conference, Miami, USA, May 2009.

${ }^{15}$ Dunn, M. H. and Tinetti, A. F., "Aeroacoustic Scattering Via the Equivalent Source Method," AIAA Paper 2004-2937, 10th AIAA/CEAS Aeroacoustics Conference, Manchester, United Kingdom, May 2004.

${ }^{16}$ Redonnet, S., Manoha, E. and Sagaut, P. "Numerical Simulation of Propagation of Small Perturbations Interacting with Flows and Solid Bodies," AIAA Paper 2001-2223, 7th CEAS/AIAA Aeroacoustics Conference, Maastricht, The Netherlands, May 2001.

${ }^{17}$ Redonnet, S., Desquesnes, G. and Manoha, E., "Numerical Study of Acoustic Installation Effects with a Computational Aeroacoustics Method", AIAA Journal, Vol. 48, No. 5, May 2010.

${ }^{18}$ Redonnet, S., Mincu, C., Manoha, E., Caruelle, B. and Sengissen, A., "Computational Aeroacoustics of a Realistic CoAxial Engine in Subsonic and Supersonic Take-Off Conditions," AIAA Paper 2009-3240, 15th AIAA / CEAS Aeroacoustics Conference, Miami, USA, May 2009.

${ }^{19}$ Cunha, G. and Redonnet, S., "An Innovative Interpolation Technique for Aeroacoustic Hybrid Methods," to be presented at the $17^{\text {th }}$ AIAA/CEAS Aeroacoustics Conference, Portland, Oregon, USA, June 2011.

${ }^{20}$ Lopes, L., Brentner, K. and Morris, P., "Framework for a Landing-Gear Model and Acoustic Prediction," Journal of Aircraft, Vol. 47, No. 3, 2010, pp. 763-774.

${ }^{21}$ Brès, G., Wessels, M. and Noelting, S., "Tandem Cylinder Noise Predictions Using Lattice Boltzmann and Ffowcs Williams-Hawkings Methods," AIAA Paper 2010-3791, 16th AIAA/CEAS Aeroacoustics Conference, Stockholm, Sweden, June 2010 . 